\title{
Nonstandard signatures of vectorlike quarks in a leptophobic 221 model
}

\author{
Kasinath Das, ${ }^{*}$ Tanmoy Mondal, ${ }^{\dagger}$ and Santosh Kumar Rai ${ }^{\ddagger}$ \\ Regional Centre for Accelerator-based Particle Physics, Harish-Chandra Research Institute, \\ HBNI, Chhatnag Road, Jhusi, Allahabad 211019, India
}

(Received 13 February 2019; published 4 June 2019)

\begin{abstract}
We consider vectorlike quarks in a leptophobic 221 model characterized by the gauge group $S U(2)_{L} \times S U(2)_{2} \times U(1)_{X}$, where the $S U(2)_{2}$ is leptophobic in nature. We discuss the pattern of mixing between Standard Model quarks and vectorlike quarks and how we prevent tree level flavor-changing interactions in the model. The model also predicts tauphilic scalars decaying mostly to tau leptons. We consider a typical signal of the model in the form of pair production of top-type vectorlike quarks which decays to the tauphilic scalars and a third generation quark. We analyze the resulting final state signal for the $13 \mathrm{TeV}$ LHC, containing $\geq 3 j(1 b)+\geq 2 \tau+\geq 1 l$ and discuss the discovery prospects of such vectorlike quarks with nonstandard decay modes.
\end{abstract}

DOI: 10.1103/PhysRevD.99.115002

\section{INTRODUCTION}

As the Large Hadron Collider (LHC) churns out more and more data and gets ready for an energy upgrade, lack of new physics signal at the high energy frontier only makes us more intrigued with what picture of beyond Standard Model (SM) could eventually emerge. The SM itself highlights the great success of gauge theory and a natural extension would be in the form of additional gauge symmetries with new matter fields. We know that all the three generations of matter fields in the SM are chiral in nature. The possibility of a fourth generation of chiral fermions, especially quarks has been excluded by the Higgs signal strength measurements along with the electroweak precision data [1]. However the possibility of having vectorlike quarks (VLQ) whose left- and right-chiral components transform in the same way under the SM gauge group still exists and are being searched for at the LHC.

The collider signatures of a VLQ depends on its possible decay modes. The existing searches for VLQs are under the assumption that they decay to a SM boson and a SM quark. For example searches on toplike VLQ with electric charge $Q=+\frac{2}{3}$ assume that it decays to $Z t, W^{+} b$ and $h t$ and for a bottomlike VLQ with electric charge $Q=-\frac{1}{3}$, the decay

\footnotetext{
*kasinath.das91@gmail.com

tanmoymondal@hri.res.in

*skrai@hri.res.in
}

Published by the American Physical Society under the terms of the Creative Commons Attribution 4.0 International license. Further distribution of this work must maintain attribution to the author(s) and the published article's title, journal citation, and DOI. Funded by SCOAP. modes are $Z b, W^{-} t$ and $h b$. Assuming strong pair production, both ATLAS and CMS collaborations have obtained different lower limits on the masses of third generation VLQs for different branching ratio hypotheses [2-7]. The most stringent lower bound on the mass of a toplike VLQ obtained by using the LHC Run 2 data is $1.3 \mathrm{TeV}$ given by CMS [2] and $1.43 \mathrm{TeV}$ given by ATLAS [6] while the most stringent lower bound on the mass of a bottomlike VLQ is 1.24 TeV given by CMS [2] and $1.35 \mathrm{TeV}$ given by ATLAS [5]. Since the single production of VLQs depend on the mixing between VLQs and SM quarks, based on the searches for single-production of VLQs both CMS and ATLAS collaborations have given exclusion limits for the product of production cross section and branching fraction for different mass values [8-10]. Extensive phenomenological studies on VLQs in standard decay scenarios exist in literature [11-17].

Exotic fermions are a necessary ingredient in some gauge extended models for anomaly cancellation, e.g., exotic quarks in leptophobic 221 model $[18,19]$, exotic leptons in hadrophobic 221 model [18] and exotic fermions in almost all $U(1)^{\prime}$ extensions [20]. These exotic fermions become vectorlike once the full symmetry group breaks down to the SM gauge group. These fermions are quasichiral in nature, i.e., they are vectorlike under the SM gauge group but chiral under the extra gauge group [20].

VLQs in gauge extended models can have interesting collider signatures because the rich spectrum of the model opens up nonstandard decay modes for the VLQs. In this work we have considered the collider signatures of certain non-standard decay modes of toplike VLQ in a leptophobic 221 model characterized by the gauge group $S U(2)_{1} \times S U(2)_{2} \times U(1)_{X}$. Because of the presence of the nonstandard decay modes the existing 
constraints on the masses of VLQs will get significantly relaxed and the VLQ can lie at the sub-TeV scale. Phenomenological studies of VLQs having nonstandard decay modes exist in literature for various nonminimal extensions of SM [21-34]. Collider signatures of vectorlike quarks in $U(1)$ gauge extensions have been considered in [35-37].

In general, the mixing between SM quarks and VLQs which play an important role in the phenomenology of VLQs also generates tree level flavor-changing neutral current (FCNC) interactions of SM quarks with the $Z$ boson. Several studies on the mixing of VLQs with SM quarks are available in the literature [16,38-43] which take into account constraints from flavor physics and electroweak precision measurements. Mixings are strongly constrained from FCNC processes. Even in the presence of mixings with VLQs the possibility of avoiding tree level FCNC interactions is possible for judiciously chosen mixing patterns [44]. We discuss such a mixing pattern for the leptophobic 221 model which avoids tree level flavor-changing interactions with the $Z$ and $Z^{\prime}$. For general mixing scenarios between VLQs and SM quarks, all the neutral scalars present in the model have flavor-changing interactions, thus making it difficult to get a $125 \mathrm{GeV}$ neutral Higgs and simultaneously satisfying constraints from FCNC processes. We show that it is possible to avoid flavor-changing interactions for certain neutral scalars (non-FCNH scalars), which can then lie at sub-TeV scale. In this work we study the collider signatures of the third generation top-type VLQ decaying to a final state with one of these non-FCNH scalars (other than the $125 \mathrm{GeV}$ Higgs) and a third generation SM quark and the scalars then decay dominantly to tau leptons.

The paper is organized as follows. In Sec. II we discuss our model. In Sec. III we discuss about the interaction of the VLQs with the SM gauge bosons. In Sec. IV we discuss the pattern of mixing between the SM quarks and the VLQs for which FCNC interactions of SM quarks with Z-boson and the FCNH interactions with the SM-Higgs boson is zero at the tree level. In Sec. V we discuss the possible phenomenology of VLQs in the model and explore the possible collider signatures in Sec. VI. Finally we conclude and summarize in Sec. VII.

\section{THE MODEL}

The $S U(2)$ extensions of the SM characterized by the gauge group $S U(3)_{C} \times S U(2)_{1} \times S U(2)_{2} \times U(1)_{X}$ are generally called 221 models in the literature. Depending on the way the SM fermions transform under the gauge groups, different versions of 221 models are possible, viz. leptophobic, hadrophobic and left-right symmetric etc. The model is called leptophobic when the SM right-chiral leptons are singlets under $S U(2)_{2}$, hadrophobic when the right-chiral SM quarks are singlets under $S U(2)_{2}$ and leftright symmetric when both the SM right-chiral quarks and the right-chiral leptons (with the addition of right-chiral neutrinos to the model) form doublets under $S U(2)_{2}$. The most popular among all 221 models is the left-right symmetric model [45-50]. A general classification of all 221 models has been done in [18] based on two types of symmetry breaking patterns of the gauge group. The two patterns are as follows:

(i) Type-I: $S U(2)_{1}$ is identified with $S U(2)_{L}$ of $\mathrm{SM}$. The first stage of symmetry breaking is $S U(2)_{2} \times U(1)_{X} \rightarrow U(1)_{Y}$. The second stage of symmetry breaking is $S U(2)_{L} \times U(1)_{Y} \rightarrow U(1)_{e m}$.

(ii) Type-II: $U(1)_{X}$ is identified with $U(1)_{Y}$ of SM. The first stage of symmetry breaking is $S U(2)_{1} \times$ $S U(2)_{2} \rightarrow S U(2)_{L}$. The second stage of symmetry breaking is $S U(2)_{L} \times U(1)_{Y} \rightarrow U(1)_{e m}$.

Certain type of 221 models need exotic fermions for the cancellation of anomalies. For example, the leptophobic 221 model needs exotic quarks and the hadrophobic 221 model requires exotic leptons. These exotic fermions become vectorlike after the breaking of the full symmetry group down to SM gauge group.

In this work we consider the leptophobic 221 model, which follows the type-I symmetry breaking pattern described above and the SM leptons are singlets under the $S U(2)_{2}$ gauge group. ${ }^{1}$ We will denote $S U(2)_{1}$ as $S U(2)_{L}$ throughout the article. The scalar sector of the model contains two scalar doublets represented by $H_{1}$ and $H_{2}$, and a bidoublet scalar represented by $\Phi$. The symmetry breaking of the full gauge group to $U(1)_{e m}$ by the scalars occurs in two stages:

$$
\begin{aligned}
& \text { Stage I: } S U(2)_{2} \times U(1)_{X} \stackrel{\left\langle H_{2}\right\rangle}{\longrightarrow} U(1)_{Y}, \\
& \text { Stage II: } S U(2)_{L} \times U(1)_{Y} \stackrel{\langle\Phi\rangle,\left\langle H_{1}\right\rangle}{\longrightarrow} U(1)_{e m} .
\end{aligned}
$$

The first stage of symmetry breaking of the full gauge group to the $\mathrm{SM}$ gauge group $\left(S U(2)_{L} \times U(1)_{Y}\right)$ is achieved by the vacuum expectation value (VEV) of $\mathrm{H}_{2}$, which is a doublet under $S U(2)_{2}$. The subsequent second stage breaking of the SM gauge group to the $U(1)_{e m}$ gauge group occurs, once $\Phi$, a bidoublet under $S U(2)_{L} \times S U(2)_{2}$ or $H_{1}$, a doublet under $S U(2)_{L}$ obtains a VEV. The fermion and scalar field content of the model and their corresponding charges under the symmetry group are listed in Table I. The left chiral fields $Q_{i L}$ and $L_{i L}$ transform as doublets under $S U(2)_{L}$ and are identical to the left chiral fields of the SM transforming under the $S U(2)_{L}$. The right handed quarks $\left(u_{i R}^{0}, d_{i R}^{0}\right)$ form doublets under the gauge group $S U(2)_{2}$ as it is in case of left-right symmetric model. But unlike the left-right symmetric model for this version of

\footnotetext{
${ }^{1}$ The model has been considered previously in [19] to explain the reported excess for a narrow width resonance around $2 \mathrm{TeV}$ in the $W Z, W W$, and $Z Z$ channel by the ATLAS collaboration [51] using the $20.3 \mathrm{fb}^{-1}$ of data of $8 \mathrm{TeV}$ LHC.
} 
TABLE I. Fields and their corresponding charges under the gauge group $S U(3)_{C} \times S U(2)_{1} \times S U(2)_{2} \times U(1)_{X}$. The model contains three generations of fermions for $i=1,2,3$.

\begin{tabular}{lccc}
\hline \hline$Q_{i L}=\left(\begin{array}{c}u_{i L}^{0} \\
d_{i L}^{0}\end{array}\right)$ & $\left(3,2,1, \frac{1}{6}\right)$ & $Q_{i R}=\left(\begin{array}{c}u_{i R}^{0} \\
d_{i R}^{0}\end{array}\right)$ & $\left(3,1,2, \frac{1}{6}\right)$ \\
$X Q_{i L}=\left(\begin{array}{c}x u_{i L}^{0} \\
x d_{i L}^{0}\end{array}\right)$ & $\left(3,1,2, \frac{1}{6}\right)$ & $x u_{i R}^{0}$ & $\left(3,1,1, \frac{2}{3}\right)$ \\
$L_{i L}=\left(\begin{array}{c}\nu_{L} \\
e_{i L}\end{array}\right)$ & $\left(1,2,1,-\frac{1}{2}\right)$ & $x d_{i R}^{0}$ & $\left(3,1,1,-\frac{1}{3}\right)$ \\
$\Phi$ & $(1,2,2,0)$ & $e_{i R}$ & $(1,1,1,-1)$ \\
& & $H_{1}$ & $\left(1,2,1,-\frac{1}{2}\right)$ \\
& & $H_{2}$ & $\left(1,1,2,-\frac{1}{2}\right)$ \\
\hline \hline
\end{tabular}

a 221 model there are no lepton doublets under the $S U(2)_{2}$ gauge group and hence the $S U(2)_{2}$ is leptophobic in nature. Therefore the model contains exotic quarks to ensure triangle anomaly cancellation. Each generation of the exotic quark sector of the model contains a field $X Q_{i L}$ formed out of two left chiral fields $x u_{i L}^{0}$ and $x d_{i L}^{0}$, and transforms as a doublet under the $S U(2)_{2}$ gauge group. For each generation the model also contains two right chiral fields $x u_{i R}^{0}$ and $x d_{i R}^{0}$ which are singlets under both $S U(2)_{L}$ and $S U(2)_{2}$. The right handed leptons $e_{i R}$ transform as singlets under both the $S U(2)$ gauge groups.

Note that the exotic quarks present in the model are chiral in nature under the full unbroken gauge group. However, after the stage-I symmetry breaking $S U(2)_{2} \times U(1)_{X} \rightarrow U(1)_{Y}$, the exotic quarks become vectorlike under the $S U(2)_{L} \times U(1)_{Y}$, which is the SM gauge group. This feature can be realized by following the definition of hypercharge quantum number $Y$ after the first stage of symmetry breaking which is given by

$$
Y=T_{2_{3}}+Q_{X},
$$

where $T_{23}$ denote the diagonal generator of $S U(2)_{2}$ and $Q_{X}$ represents the charge for the gauge group $U(1)_{X}$. The hypercharge quantum number for the exotic quarks are given by $Y\left(x u_{L}^{0}\right)=\frac{2}{3}=Y\left(x u_{R}^{0}\right)$ and $Y\left(x d_{L}^{0}\right)=-\frac{1}{3}=$ $Y\left(x d_{R}^{0}\right)$, i.e., they are vectorlike with respect to the $S U(2)_{L} \times U(1)_{Y}$ gauge group.

After the stage II of symmetry breaking, the electric charge for a field is given by

$$
Q=T_{1_{3}}+Y
$$

where $T_{13}$ denote the diagonal generator of $S U(2)_{1}$. Following the above definition the electric charges for the VLQs are given by $Q\left(x u_{i}^{0}\right)=+\frac{2}{3}$ and $Q\left(x d_{i}^{0}\right)=-\frac{1}{3}$.

\section{A. Yukawa sector}

The Yukawa Lagrangian including the bilinear mass terms for the model is given by

$$
\begin{aligned}
-L_{\text {Yukawa }}= & Y_{i j}^{q} \overline{Q_{i L}} \Phi Q_{j R}+Y_{i j}^{q C} \overline{Q_{i L}} \tilde{\Phi} Q_{j R} \\
& +Y_{i j}^{x q u} \overline{X Q_{i L}} x u_{j R}^{0} H_{2}-Y_{i j}^{x q x d} \overline{X Q_{i L}} x d_{j_{R}}^{0} \widetilde{H_{2}} \\
& +Y_{i j}^{q x u} \overline{Q_{i L}} x u_{j R}^{0} H_{1}-Y_{i j}^{q x d} \overline{Q_{i L}} x d_{j R}^{0} \widetilde{H_{1}} \\
& +\mu_{i j} \overline{X Q_{i L}} Q_{j_{R}}+Y_{i j}^{L} \overline{L_{i L}} e_{j R} \widetilde{H_{1}}+\text { H.c. },
\end{aligned}
$$

where $i, j=1,2,3$ and we define the fields,

$$
\begin{aligned}
\tilde{\Phi} & =\sigma_{2} \Phi^{*} \sigma_{2} \equiv(1,2,2,0), \quad \widetilde{H_{1}}=i \sigma_{2} H_{1}^{*} \equiv\left(1,2,1, \frac{1}{2}\right), \\
\widetilde{H_{2}} & =i \sigma_{2} H_{2}^{*} \equiv\left(1,1,2, \frac{1}{2}\right) .
\end{aligned}
$$

The $Y$ matrices in the above Lagrangian are Yukawa couplings. Note that after the scalars get VEVs the $Y_{i j}^{q}$ and $Y_{i j}^{q C}$ terms will give masses to the SM quarks while the $Y_{i j}^{x q x u}$ and $Y_{i j}^{x q x d}$ terms give masses to the VLQs. The terms in the Lagrangian containing $Y_{i j}^{q x u}, Y_{i j}^{q x d}$ and the bilinear term with $\mu_{i j}$ will generate mixing between the SM quarks and the VLQs. Since the model does not contain any lepton doublet under $S U(2)_{2}$ gauge group, the charged leptons will get mass from the VEV of the doublet $H_{1}$ unlike the quarks which get their mass from the bidoublet $\Phi$.

\section{B. Scalar sector}

The tree level scalar potential for the model in terms of a complete set of linearly independent gauge invariant terms is given by

$$
\begin{aligned}
V= & -\mu_{1}^{2} \operatorname{Tr}\left[\Phi^{\dagger} \Phi\right]-\left\{\mu_{2}^{2} \operatorname{Tr}\left[\tilde{\Phi}^{\dagger} \Phi\right]+\mu_{2}^{2 *} \operatorname{Tr}\left[\Phi^{\dagger} \tilde{\Phi}\right]\right\}-\mu_{3}^{2} H_{1}^{\dagger} H_{1}-\mu_{4}^{2} H_{2}^{\dagger} H_{2}+\left\{M_{1} H_{1}^{\dagger} \Phi H_{2}+M_{1}^{*} H_{2}^{\dagger} \Phi^{\dagger} H_{1}\right\} \\
& +\left\{M_{2} H_{1}^{\dagger} \tilde{\Phi} H_{2}+M_{2}^{*} H_{2}^{\dagger} \tilde{\Phi}^{\dagger} H_{1}\right\}+\lambda_{1}\left\{\operatorname{Tr}\left[\Phi^{\dagger} \Phi\right]\right\}^{2}+\left\{\lambda_{2}\left\{\operatorname{Tr}\left[\Phi^{\dagger} \tilde{\Phi}\right]\right\}^{2}+\lambda_{2}^{*}\left\{\operatorname{Tr}\left[\tilde{\Phi}^{\dagger} \Phi\right]\right\}^{2}\right\}+\lambda_{3} \operatorname{Tr}\left[\Phi^{\dagger} \tilde{\Phi}\right] \operatorname{Tr}\left[\tilde{\Phi}^{\dagger} \Phi\right] \\
& +\left\{\lambda_{4} \operatorname{Tr}\left[\Phi^{\dagger} \Phi\right] \operatorname{Tr}\left[\Phi^{\dagger} \tilde{\Phi}\right]+\lambda_{4}^{*} \operatorname{Tr}\left[\Phi^{\dagger} \Phi\right] \operatorname{Tr}\left[\tilde{\Phi}^{\dagger} \Phi\right]\right\}+\beta_{1} \operatorname{Tr}\left[\Phi^{\dagger} \Phi\right]\left(H_{1}^{\dagger} H_{1}\right)+\left\{\beta_{2} \operatorname{Tr}\left[\tilde{\Phi}^{\dagger} \Phi\right]\left(H_{1}^{\dagger} H_{1}\right)+\beta_{2}^{*} \operatorname{Tr}\left[\Phi^{\dagger} \tilde{\Phi}\right]\left(H_{1}^{\dagger} H_{1}\right)\right\} \\
& +\beta_{3} \widetilde{H}_{1}^{\dagger} \Phi \Phi^{\dagger} \widetilde{H}_{1}+\alpha_{1} \operatorname{Tr}\left[\Phi^{\dagger} \Phi\right]\left(H_{2}^{\dagger} H_{2}\right)+\left\{\alpha_{2} \operatorname{Tr}\left[\tilde{\Phi}^{\dagger} \Phi\right]\left(H_{2}^{\dagger} H_{2}\right)+\alpha_{2}^{*} \operatorname{Tr}\left[\Phi^{\dagger} \tilde{\Phi}\right]\left(H_{2}^{\dagger} H_{2}\right)\right\} \\
& +\alpha_{3} \widetilde{H}_{2}^{\dagger} \Phi^{\dagger} \Phi \widetilde{H}_{2}+\rho_{1}\left(H_{1}^{\dagger} H_{1}\right)^{2}+\rho_{2}\left(H_{2}^{\dagger} H_{2}\right)^{2}+\rho_{3}\left(H_{1}^{\dagger} H_{1}\right)\left(H_{2}^{\dagger} H_{2}\right) .
\end{aligned}
$$


In general the parameters from the set $\left\{\mu_{1}^{2}, \mu_{3}^{2}, \mu_{4}^{2}\right.$, $\left.\lambda_{1}, \lambda_{3}, \beta_{1}, \beta_{3}, \alpha_{1}, \alpha_{3}, \rho_{1}, \rho_{2}, \rho_{3}\right\}$ are real and others are complex. In this paper, for simplicity we have considered all the parameters to be real.

The scalar fields in component form can be written as

$$
H_{1}=\left(\begin{array}{c}
\chi^{0} \\
\chi^{-}
\end{array}\right), \quad H_{2}=\left(\begin{array}{c}
\chi^{0} \\
\chi^{\prime-}
\end{array}\right), \quad \Phi=\left(\begin{array}{cc}
\phi_{1}^{0} & \phi_{1}^{+} \\
\phi_{2}^{-} & \phi_{2}^{0}
\end{array}\right),
$$

where

$$
\begin{array}{ll}
\phi_{1}^{0}=\frac{1}{\sqrt{2}}\left(v_{1}+\phi_{1}^{0 r}+i \phi_{1}^{0 i}\right), & \phi_{2}^{0}=\frac{1}{\sqrt{2}}\left(v_{2}+\phi_{2}^{0 r}+i \phi_{2}^{0 i}\right), \\
\chi^{0}=\frac{1}{\sqrt{2}}\left(v_{3}+\chi^{0 r}+i \chi^{0 i}\right), & \chi^{00}=\frac{1}{\sqrt{2}}\left(u+\chi^{\prime 0 r}+i \chi^{\prime 0 i}\right) .
\end{array}
$$

The structure of VEVs for the scalar fields are

$$
\begin{aligned}
\left\langle H_{1}\right\rangle & =\frac{1}{\sqrt{2}}\left(\begin{array}{c}
v_{3} \\
0
\end{array}\right), \quad\left\langle H_{2}\right\rangle=\frac{1}{\sqrt{2}}\left(\begin{array}{l}
u \\
0
\end{array}\right), \\
\langle\Phi\rangle & =\frac{1}{\sqrt{2}}\left(\begin{array}{cc}
v_{1} & 0 \\
0 & v_{2}
\end{array}\right) .
\end{aligned}
$$

The set of tadpole equations $\left\{\frac{\partial V}{\partial \phi_{1}^{0 r}}=0, \frac{\partial V}{\partial \phi_{2 r}^{0 r}}=0\right.$, $\left.\frac{\partial V}{\partial \chi^{0 r}}=0, \frac{\partial V}{\partial \chi^{0 r}}=0\right\}$ have been solved in terms of $\mu_{1}^{2}, \mu_{2}^{2}$, $\mu_{3}^{2}, \mu_{4}^{2}$ and are given in the Appendix A. The components of the mass square matrices for the $C P$ even scalars $\left(M_{S}^{2}\right)$ in the $\left(\phi_{1}^{0 r}, \phi_{2}^{0 r}, \chi^{\prime 0 r}, \chi^{0 r}\right)$ basis, for the $C P$ odd scalars $\left(M_{P}^{2}\right)$ in the $\left(\phi_{1}^{0 i}, \phi_{2}^{0 i}, \chi^{10 i}, \chi^{0 i}\right)$ basis and for the charged scalars $\left(M_{C}^{2}\right)$ in the $\left(\phi_{1}^{+}, \phi_{2}^{+}, \chi^{\prime+}, \chi^{+}\right)$basis are given in Appendix B 1, B 2 and B 3 respectively.

There are four physical $C P$ even scalars in the model. Two of the four $C P$ odd scalars will be physical and the other two are massless Goldstone bosons which become part of the two massive neutral gauge bosons. Similarly there will be two physical charged scalars and the other two Goldstone bosons become part of the two massive charged gauge bosons. Hence, after the spontaneous symmetry breaking followed by the Higgs mechanism, the physical spectrum of the scalar sector consists of four neutral $C P$ even scalars, two neutral $C P$ odd scalars and two charged scalars (and their antiparticles).

\section{Gauge boson sector}

The gauge couplings corresponding to the gauge groups $S U(2)_{L}, S U(2)_{2}$ and $U(1)_{X}$ are respectively represented by $g_{1}, g_{2}$ and $g_{X}$. Based on the two stages of symmetry breaking pattern we define two mixing angles $\phi$ and $\theta_{W}$ in terms of which the gauge couplings are given by $g_{1}=\frac{e}{\sin \theta_{W}}, \quad g_{2}=\frac{e}{\cos \theta_{W} \sin \phi}, \quad g_{X}=\frac{e}{\cos \theta_{W} \cos \phi}$.

After the stage I of symmetry breaking $S U(2)_{2} \times U(1)_{X} \rightarrow$ $U(1)_{Y}$ the gauge group becomes the Standard Model gauge group $S U(2)_{L} \times U(1)_{Y}$. The $\mathrm{SM}$ hypercharge gauge coupling $g_{Y}$ for the gauge group $U(1)_{Y}$ is given by the relation $\frac{1}{g_{Y}^{2}}=\frac{1}{g_{2}^{2}}+\frac{1}{g_{X}^{2}}$. With the stage II of symmetry breaking $S U(2)_{1} \times U(1)_{Y} \rightarrow U(1)_{e m}$ the electromagnetic gauge coupling constant $e$ is defined by $\frac{1}{e^{2}}=\frac{1}{g_{1}^{2}}+\frac{1}{g_{Y}^{2}}$. Here the angle $\theta_{W}$ denotes the weak mixing angle in the SM. At the end of the two stages of symmetry breaking the electromagnetic charge for any field in the model is defined by $Q=T_{1_{3}}+T_{2_{3}}+Q_{X}$.

The gauge bosons corresponding to the gauge groups are denoted by:

$S U(2)_{1}: W_{1, \mu}^{ \pm}, W_{1, \mu}^{3} ; \quad S U(2)_{2}: W_{2, \mu}^{ \pm}, W_{2, \mu}^{3} ; \quad U(1)_{X}: X_{\mu}$.

The mass square matrix for the charged gauge boson sector in the $\left(W_{1, \mu}^{ \pm}, W_{2, \mu}^{ \pm}\right)$basis is given by

$M_{W_{1}-W_{2}}^{2}=\frac{1}{4}\left(\begin{array}{cc}g_{1}^{2}\left(v_{1}^{2}+v_{2}^{2}+v_{3}^{2}\right) & -2 g_{1} g_{2} v_{1} v_{2} \\ -2 g_{1} g_{2} v_{1} v_{2} & g_{2}^{2}\left(u^{2}+v_{1}^{2}+v_{2}^{2}\right)\end{array}\right)$.

Since the vacuum expectation value $v_{3}$ gives masses to the charged leptons, for simplicity we consider the situation where $v_{1}^{2}+v_{2}^{2} \gg v_{3}^{2}$ and to have the stage II breaking at a higher scale we consider $u \gg v_{1}, v_{2}, v_{3}$. Based on this we define a small parameter $\epsilon$ which is given by $\frac{v^{2}}{u^{2}}$, where $v=\sqrt{v_{1}^{2}+v_{2}^{2}+v_{3}^{2}} \simeq 246 \mathrm{GeV}$.

The mass eigenstates for the charged gauge boson sector in terms of the gauge eigenstates are given by

$$
\left(\begin{array}{c}
W^{ \pm} \\
W^{\prime \pm}
\end{array}\right)=\left(\begin{array}{cc}
\cos \theta_{\mathrm{ww}^{\prime}} & \sin \theta_{\mathrm{ww}^{\prime}} \\
-\sin \theta_{\mathrm{ww}^{\prime}} & \cos \theta_{\mathrm{ww}^{\prime}}
\end{array}\right)\left(\begin{array}{c}
W_{1}^{ \pm} \\
W_{2}^{ \pm}
\end{array}\right),
$$

with the mixing angle given up to order $\epsilon$ by the relation

$\cos \theta_{\mathrm{ww}^{\prime}} \simeq 1$ and $\quad \sin \theta_{\mathrm{ww}^{\prime}} \simeq \epsilon \frac{\sin \phi \sin 2 \beta}{\tan \theta_{W}}$,

with $\beta=\tan ^{-1}\left(\frac{v_{1}}{v_{2}}\right)$.

The mass eigenstate $W$ denotes the observed SM $W$ boson while the new $W^{\prime}$ is heavy with mass at $\mathrm{TeV}$ scale. The mass squared matrix for the neutral gauge boson sector in the basis $\left(W_{1}^{3}, W_{2}^{3}, X\right)$ is 
$\frac{1}{4}\left(\begin{array}{ccc}g_{1}^{2}\left(v_{1}^{2}+v_{2}^{2}+v_{3}^{2}\right) & -g_{1} g_{2}\left(v_{1}^{2}+v_{2}^{2}\right) & -g_{1} g_{X} v_{3}^{2} \\ -g_{1} g_{2}\left(v_{1}^{2}+v_{2}^{2}\right) & g_{2}^{2}\left(v_{1}^{2}+v_{2}^{2}+u^{2}\right) & -g_{2} g_{X} u^{2} \\ -g_{1} g_{X} v_{3}^{2} & -g_{2} g_{X} u^{2} & g_{X}^{2}\left(u^{2}+v_{3}^{2}\right)\end{array}\right)$,

and the mass eigenstates are given by

$$
\begin{aligned}
\left(\begin{array}{c}
Z^{\prime} \\
Z \\
A
\end{array}\right)= & \left(\begin{array}{ccc}
\cos \theta_{z z^{\prime}} & -\sin \theta_{z z^{\prime}} & 0 \\
\sin \theta_{z z^{\prime}} & \cos \theta_{z z^{\prime}} & 0 \\
0 & 0 & 1
\end{array}\right) \\
& \times\left(\begin{array}{ccc}
1 & 0 & 0 \\
0 & \cos \theta_{W} & -\sin \theta_{W} \\
0 & \sin \theta_{W} & \cos \theta_{W}
\end{array}\right) \\
& \times\left(\begin{array}{ccc}
\cos \phi & 0 & -\sin \phi \\
0 & 1 & 0 \\
\sin \phi & 0 & \cos \phi
\end{array}\right)\left(\begin{array}{c}
W_{2}^{3} \\
W_{1}^{3} \\
X
\end{array}\right)
\end{aligned}
$$

Among the mass eigenstates, $A$ denotes massless photon, $Z$ denotes the observed neutral heavy SM weak gauge boson and $Z^{\prime}$, the heavier neutral gauge boson that has mass at $\mathrm{TeV}$ scale. Up to order $\epsilon$ the $Z-Z^{\prime}$ mixing angle is given by

$$
\cos \theta_{z z^{\prime}} \simeq 1 \quad \text { and } \quad \sin \theta_{z z^{\prime}} \simeq \epsilon \frac{\sin \phi \cos ^{3} \phi}{\sin \theta_{W}}
$$

\section{INTERACTIONS OF VECTORLIKE QUARKS WITH GAUGE BOSONS}

The covariant derivative for a field determines its nature of interaction with the gauge bosons. To observe the vectorlike nature of the interaction of exotic quarks with the SM gauge bosons we write those terms from the covariant derivative which contains neutral gauge bosons and that is given by

$$
\begin{aligned}
g_{1} T_{1_{3}} W_{1 \mu}^{3}+g_{2} T_{2_{3}} W_{2 \mu}^{3}+g_{X} Q_{x} X_{\mu}= & e Q A_{\mu}+\frac{e}{\sin \theta_{W} \cos \theta_{W}}\left\{\left(T_{1_{3}}-Q \sin ^{2} \theta_{W}\right)+\epsilon \cos ^{2} \phi\left(\left(T_{1_{3}}-Q\right) \sin ^{2} \phi+T_{2_{3}}\right)\right\} Z_{\mu} \\
& +\frac{e}{\sin \theta_{W} \cos \theta_{W}}\left\{\left(\left(T_{1_{3}}-Q\right) \sin ^{2} \phi+T_{2_{3}}\right) \frac{\sin \theta_{W}}{\sin \phi \cos \phi}+\epsilon\left(Q \sin ^{2} \theta_{W}-T_{1_{3}}\right) \frac{\sin \phi \cos ^{3} \phi}{\sin \theta_{W}}\right\} Z_{\mu}^{\prime} .
\end{aligned}
$$

By observing the term with $Z_{\mu}$ from Eq. (18) we can conclude that the interaction strength of the left chiral fields $x u_{i L}^{0}\left(x d_{i L}^{0}\right)$ with $Z$ boson differs from the interaction strength for the right chiral fields $x u_{i R}^{0}\left(x d_{i R}^{0}\right)$ by a term proportional to $\epsilon$. This is because the left chiral and the right chiral fields do not have same $T_{23}$ value. Hence the interactions of the BSM quarks with the $Z$ boson are vectorlike in the limit $\epsilon \rightarrow 0$. The origin of the $\epsilon$ term is due to the $Z-Z^{\prime}$ mixing. Since we are interested in the situation where $u \gg v$ and hence $\epsilon=\frac{v^{2}}{u^{2}} \ll 1$, we have identified the BSM exotic quarks $\left(x u_{i}^{0}\right.$ and $\left.x d_{i}^{0}\right)$ as VLQs throughout the article.

Since the exotic quarks are singlets under $S U(2)_{L}$ the interaction strength of the exotic quarks with the $W$ boson will be very small for small values of $W-W^{\prime}$ mixing angle.

\section{FCNC IN THE PRESENCE OF VECTORLIKE QUARKS}

In SM there is no flavor-changing neutral current (FCNC) interactions of quarks with the $Z$ boson at the tree level because the quarks with same electric charge have universal charge assignments under the SM gauge group. But in models with VLQs this scenario of having universal charges under the gauge group of the model having the same electric charge breaks down. Hence the mixing between the SM quarks and the VLQs can generate tree level FCNC interactions for the SM quarks.

In our model this mixing is generated by the terms proportional to $Y_{i j}^{q x d}, Y_{i j}^{q x u}$ and $\mu_{i j}$ in the Yukawa Lagrangian in Eq. (4). For models with vectorlike quarks the possibility of restricting tree level FCNC interactions exists for special choice of mixing patterns between the quarks and VLQs [44]. It has been shown in [44] that if one linear combination of VLQs mix with only one SM quark mass eigenstate then there will be no $Z$-boson mediated FCNC interaction at tree level. This would imply that each VLQ will have a corresponding SM quark (mass eigenstate) with which it mixes. We use this formalism [44] and discuss the scenario in which the FCNC interactions vanish for our model.

The $6 \times 6$ dimensional mass matrices for the up-quark sector and for the down-quark sector are respectively given by

$$
\mathcal{M}^{u}=\left(\begin{array}{cc}
M^{u} & Y^{q x u} \frac{v_{3}}{\sqrt{2}} \\
\mu & Y^{x q x u} \frac{u}{\sqrt{2}}
\end{array}\right) \text { and } \mathcal{M}^{d}=\left(\begin{array}{cc}
M^{d} & Y^{q x d} \frac{v_{3}}{\sqrt{2}} \\
\mu & Y^{x q x} \frac{u}{\sqrt{2}}
\end{array}\right) .
$$


The matrices $Y^{q x u}, Y^{q x d}, Y^{x q x d}, \mu$ are $3 \times 3$ dimensional whose components are formed out of the Yukawa couplings in Eq. (4). The $3 \times 3$ matrices $M^{u}$ and $M^{d}$ are given by

$$
\begin{aligned}
M^{u}{ }_{i j} & =\frac{1}{\sqrt{2}}\left(Y_{i j}^{q} v_{1}+Y_{i j}^{q C} v_{2}\right) \\
M^{d}{ }_{i j} & =\frac{1}{\sqrt{2}}\left(Y_{i j}^{q} v_{2}+Y_{i j}^{q C} v_{1}\right) .
\end{aligned}
$$

The quark gauge eigenstates $\left(\hat{\mathcal{U}}_{L / R}, \hat{\mathcal{D}}_{L / R}\right)$ and the mass eigenstates $\left(\mathcal{U}_{L / R}, \mathcal{D}_{L / R}\right)$ are represented by

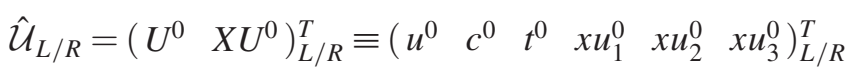

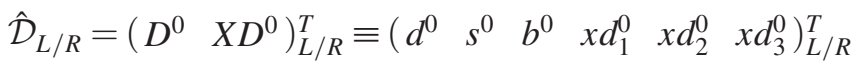
$\mathcal{U}_{L / R}=\left(\begin{array}{lllllll}U & X U\end{array}\right)_{L / R}^{T} \equiv\left(\begin{array}{llllll}u & c & t & x u_{1} & x u_{2} & x u_{3}\end{array}\right)_{L / R}^{T}$ $\mathcal{D}_{L / R}=\left(\begin{array}{lllllll}D & X D\end{array}\right)_{L / R}^{T} \equiv\left(\begin{array}{llllll}d & s & b & x d_{1} & x d_{2} & x d_{3}\end{array}\right)_{L / R}^{T}$.

The matrices $\mathcal{M}^{u}$ and $\mathcal{M}^{d}$ from Eq. (19) will be diagonalized by biunitary transformations and are given by

$$
\hat{\mathcal{U}}_{L / R}=S_{L / R}^{u} \mathcal{U}_{L / R} \quad \hat{\mathcal{D}}_{L / R}=S_{L / R}^{d} \mathcal{D}_{L / R},
$$

where the $S_{L}^{u}$ and $S_{L}^{d}$ are $6 \times 6$ unitary matrices and can be represented by

$$
\mathcal{S}_{L}^{u}=\left(\begin{array}{cc}
A_{L}^{u} & E_{L}^{u} \\
F_{L}^{u} & G_{L}^{u}
\end{array}\right), \quad \mathcal{S}_{L}^{d}=\left(\begin{array}{cc}
A_{L}^{d} & E_{L}^{d} \\
F_{L}^{d} & G_{L}^{d}
\end{array}\right)
$$

The matrices $\mathcal{S}_{R}^{u}$ and $\mathcal{S}_{R}^{d}$ can be obtained from Eq. (23) by replacing $L \rightarrow R$. The matrices $A, E, F, G$ are $3 \times 3$ dimensional and where $E$ and $F$ connect the SM quarks with the VLQs. To avoid FCNC at the tree level we choose the mixing pattern such that the matrices for the left chiral sector take the form

$$
A_{L}^{u}=\widehat{A_{L}^{u}} C_{L}^{u}, \quad F_{L}^{u}=S_{L}^{u}, \quad G_{L}^{u}=C_{L}^{u}, \quad E_{L}^{u}=-\widehat{A_{L}^{u}} S_{L}^{u}
$$

where

$$
\begin{aligned}
{\widehat{A_{L}^{u}} \dagger}^{\dagger} \widehat{A_{L}^{u}} & =\widehat{A_{L}^{u}}{\widehat{A_{L}^{u}}}^{\dagger}=\mathbb{1}, \\
C_{L}^{u} & =\operatorname{diag}\left(\cos \theta_{L}^{u}, \cos \theta_{L}^{c}, \cos \theta_{L}^{t}\right), \\
S_{L}^{u} & =\operatorname{diag}\left(\sin \theta_{L}^{u}, \sin \theta_{L}^{c}, \sin \theta_{L}^{t}\right),
\end{aligned}
$$

then,

$$
\begin{aligned}
& u_{L}=\cos \theta_{L}^{u}\left({\widehat{A_{L}^{u}}}^{\dagger}\left(\begin{array}{c}
u^{0} \\
c^{0} \\
t^{0}
\end{array}\right)_{L}\right)_{1}+\sin \theta_{L}^{u} x u_{1 L}^{0}, \\
& x u_{1 L}=-\sin \theta_{L}^{u}\left({\widehat{A_{L}^{u}} \dagger}^{\dagger}\left(\begin{array}{c}
u^{0} \\
c^{0} \\
t^{0}
\end{array}\right)_{L}\right)_{1}+\cos \theta_{L}^{u} x u_{1 L}^{0} \text {, } \\
& c_{L}=\cos \theta_{L}^{c}\left(\widehat{A}_{L}^{\dagger}\left(\begin{array}{c}
u^{0} \\
c^{0} \\
t^{0}
\end{array}\right)_{L}\right)_{2}+\sin \theta_{L}^{c} x u_{2 L}^{0}, \\
& x u_{2 L}=-\sin \theta_{L}^{c}\left({\widehat{A_{L}^{u}} \dagger}^{\dagger}\left(\begin{array}{c}
u^{0} \\
c^{0} \\
t^{0}
\end{array}\right)_{L}\right)_{2}+\cos \theta_{L}^{c} x u_{2 L}^{0}, \\
& t_{L}=\cos \theta_{L}^{t}\left(\widehat{A}_{L}^{\dagger}\left(\begin{array}{c}
u^{0} \\
c^{0} \\
t^{0}
\end{array}\right)_{L}\right)_{3}+\sin \theta_{L}^{t} x u_{3 L}^{0} \\
& x u_{3 L}=-\sin \theta_{L}^{t}\left({\widehat{A_{L}^{u}} \dagger}^{\dagger}\left(\begin{array}{c}
u^{0} \\
c^{0} \\
t^{0}
\end{array}\right)_{L}\right)_{3}+\cos \theta_{L}^{t} x u_{3 L}^{0} \text {. }
\end{aligned}
$$

From Eq. (26) it can be seen that each vectorlike quark mixes with only one linear combination of the SM gauge eigenstate quarks. The different linear combinations with which different VLQs mix are characterized by the unitary matrix $\widehat{A_{L}^{u}}$.

Similarly to avoid FCNC in the down-type quark sector we choose the mixing matrices for the left chiral down-type quarks in a similar way as above with up-types changed with down-type quarks:

$A_{L}^{d}=\widehat{A_{L}^{d}} C_{L}^{d}, \quad F_{L}^{d}=S_{L}^{d}, \quad G_{L}^{d}=C_{L}^{d}, \quad E_{L}^{d}=-\widehat{A_{L}^{d}} S_{L}^{d}$

where

$$
\begin{aligned}
{\widehat{A_{L}^{d}}}^{\dagger} \widehat{A_{L}^{d}} & =\widehat{A_{L}^{d}}{\widehat{A_{L}^{d}}}^{\dagger}=\mathbb{1}, \\
C_{L}^{d} & =\operatorname{diag}\left(\cos \theta_{L}^{d}, \cos \theta_{L}^{s}, \cos \theta_{L}^{b}\right), \\
S_{L}^{d} & =\operatorname{diag}\left(\sin \theta_{L}^{d}, \sin \theta_{L}^{s}, \sin \theta_{L}^{b}\right) .
\end{aligned}
$$

The mixing matrices and the mass eigenstates for the righthanded fields can be obtained by replacing $L \rightarrow R$ in Eqs. (24)-(28). Note that both Eq. (26) and its right handed counterpart show that in the absence of mixing between the SM quarks and the VLQs, the unitary matrices $\widehat{A_{L}^{u}}$ and $\widehat{A_{R}^{u}}$ are the matrices which diagonalize the mass matrix for the up-quark sector of the SM. The same can be concluded for the down-quark sector also. 


\section{A. CKM matrix in presence of vectorlike quarks}

The interaction of the SM left-chiral gauge eigenstates with the $W$ boson is given by

$$
\begin{aligned}
& \frac{g_{1}}{\sqrt{2}} W_{\mu}^{+} \overline{U_{L}^{0}} \gamma^{\mu} D_{L}^{0}=\frac{g_{1}}{\sqrt{2}} W_{\mu}^{+}\left\{\overline{U_{L}} A_{L}^{u \dagger} A_{L}^{d} \gamma^{\mu} D_{L}\right. \\
& +\overline{X U_{L}} E_{L}^{u \dagger} A_{L}^{d} \gamma^{\mu} D_{L}+\overline{U_{L}} A_{L}^{u \dagger} E_{L}^{d} \gamma^{\mu} X D_{L} \\
& \left.+\overline{X U_{L}} E_{L}^{u \dagger} E_{L}^{d} \gamma^{\mu} X D_{L}\right\} \text {. }
\end{aligned}
$$

Based on the interactions of the SM quark mass eigenstates with the $W$ boson the Cabibbo-Kobayashi-Maskawa (CKM) matrix is defined as

$$
V_{L}^{\mathrm{CKM}}=A_{L}^{u \dagger} A_{L}^{d}=C_{L}^{u} \widehat{A}_{L}^{\dagger} \widehat{A}_{L}^{d} C_{L}^{d} .
$$

$V_{L}^{\mathrm{CKM}}$ corresponds to the measured CKM matrix. It can be noted that in the presence of mixing between SM quarks and VLQs the matrix $V_{L}^{\mathrm{CKM}}$ is not unitary. The deviation from unitarity of the measured CKM matrix will put constraints on the mixing angles contained in the matrices $C_{L}^{u}$ and $C_{L}^{d}$.

Similarly the interaction term of the SM right-chiral quark gauge eigenstates with the $W^{\prime}$ gauge boson is given by

$$
\begin{aligned}
\frac{g_{2}}{\sqrt{2}} W_{\mu}^{\prime+} \overline{U_{R}^{0}} \gamma^{\mu} D_{R}^{0}= & \frac{g_{1}}{\sqrt{2}} W_{\mu}^{\prime+}\left\{\overline{U_{R}} A_{R}^{u \dagger} A_{R}^{d} \gamma^{\mu} D_{R}\right. \\
& +\overline{X U_{R}} E_{R}^{u \dagger} A_{R}^{d} \gamma^{\mu} D_{R}+\overline{U_{R}} A_{R}^{u \dagger} E_{R}^{d} \gamma^{\mu} X D_{R} \\
& \left.+\overline{X U_{R}} E_{R}^{u \dagger} E_{R}^{d} \gamma^{\mu} X D_{R}\right\} .
\end{aligned}
$$

We define a right-handed CKM matrix given by

$$
V_{R}^{\mathrm{CKM}}=A_{R}^{u \dagger} A_{R}^{d}=C_{R}^{u} \widehat{A}_{R}^{\dagger} \widehat{A}_{R}^{d} C_{R}^{d} .
$$

\section{B. FCNC interaction with $Z$ and $Z^{\prime}$}

To see how the choice of mixing pattern that we have considered avoids FCNC at tree level, we focus on the terms containing $Z$ boson in Eq. (18). Since all the fields $u_{L}^{0}, c_{L}^{0}$ and $t_{L}^{0}$ carry universal charges under the full gauge group, we can write their interaction with the $Z$ boson in terms of $U^{0}$ of Eq. (21) as

$$
\begin{aligned}
& \frac{e}{\sin \theta_{W} \cos \theta_{W}}\left\{\left(\frac{1}{2}-\frac{2}{3} \sin ^{2} \theta_{W}\right)-\frac{1}{6} \epsilon \cos ^{2} \phi \sin ^{2} \phi\right\} \\
& \times Z_{\mu} \overline{U_{L}^{0}} \gamma^{\mu} U_{L}^{0} .
\end{aligned}
$$

The flavor diagonal nature can be seen by writing $\overline{U_{L}^{0}} \gamma^{\mu} U_{L}^{0}$ in terms of mass eigenstates by using the Eqs. (21)-(24) and is given by

$$
\begin{aligned}
\overline{U_{L}^{0}} \gamma^{\mu} U_{L}^{0}= & \overline{U_{L}} C_{L}^{u} \gamma^{\mu} U_{L} \\
& -\left(\overline{U_{L}} C_{L}^{u} S_{L}^{u} \gamma^{\mu} X U_{L}+\overline{X U_{L}} S_{L}^{u} C_{L}^{u} \gamma^{\mu} U_{L}\right) \\
& +\overline{X U_{L}} S_{L}^{u} \gamma^{\mu} X U_{L} .
\end{aligned}
$$

Since $C_{L}^{u}$ is a diagonal matrix, the first term in the right hand side of the above equation is diagonal in the mass eigenstates $u_{L}, c_{L}$ and $t_{L}$. Similarly, with the equivalent form of Eq. (34) for $U_{R}^{0}, X U_{L / R}^{0}, D_{L / R}^{0}$ and $X D_{L / R}^{0}$, and since $S_{L}^{u}$ is also a diagonal matrix, we find that there is no flavour-changing interactions of the SM quarks with the $Z$ boson. Again, by expanding the interaction terms for $Z^{\prime}$ in Eq. (18) we find that the $Z^{\prime}$ also does not have any flavorchanging interactions with the SM quarks for the chosen mixing pattern.

\section{FCNH interaction with Higgs bosons}

The unitary matrices in Eq. (22) diagonalize the quark mass matrices, i.e.,

$\mathcal{S}_{L}^{u \dagger}\left(\begin{array}{cc}\frac{1}{\sqrt{2}}\left(Y^{q} v_{1}+Y^{q C} v_{2}^{*}\right) & Y^{q x u} \frac{v_{3}}{\sqrt{2}} \\ \mu & Y^{x q x u} \frac{u}{\sqrt{2}}\end{array}\right) \mathcal{S}_{R}^{u}=\mathcal{M}_{\mathrm{diag}}^{u}$,
$\mathcal{S}_{L}^{d \dagger}\left(\begin{array}{cc}\frac{1}{\sqrt{2}}\left(Y^{q} v_{2}+Y^{q C} v_{1}^{*}\right) & Y^{q x d} \frac{v_{3}}{\sqrt{2}} \\ \mu & Y^{x q x d} \frac{u}{\sqrt{2}}\end{array}\right) \mathcal{S}_{R}^{d}=\mathcal{M}_{\mathrm{diag}}^{d}$,

where

$$
\mathcal{M}_{\text {diag }}^{u}=\left(\begin{array}{cc}
M_{\text {diag }}^{u} & 0 \\
0 & M_{\text {diag }}^{x u}
\end{array}\right) \text { and } \mathcal{M}_{\text {diag }}^{d}=\left(\begin{array}{cc}
M_{\text {diag }}^{d} & 0 \\
0 & M_{\text {diag }}^{x d}
\end{array}\right) \text {. }
$$

And

$$
\begin{aligned}
& M_{\text {diag }}^{u}=\operatorname{diag}\left(m_{u}, m_{c}, m_{t}\right), \quad M_{\text {diag }}^{d}=\operatorname{diag}\left(m_{d}, m_{s}, m_{b}\right), \\
& M_{\text {diag }}^{x u}=\operatorname{diag}\left(m_{x u_{1}}, m_{x u_{2}}, m_{x u_{3}}\right), \\
& M_{\text {diag }}^{x d}=\operatorname{diag}\left(m_{x d_{1}}, m_{x d_{2}}, m_{x d_{3}}\right) .
\end{aligned}
$$

From Eq. (35) the Yukawa couplings with the bidoublet in terms of mixing matrices are given by

$$
\begin{aligned}
& \frac{1}{\sqrt{2}}\left(Y^{q} v_{1}+Y^{q C} v_{2}^{*}\right)=A_{L}^{u} M_{\mathrm{diag}}^{u} A_{R}^{u \dagger}+E_{L}^{u} M_{\mathrm{diag}}^{x u} E_{R}^{u \dagger}, \\
& \frac{1}{\sqrt{2}}\left(Y^{q} v_{2}+Y^{q C} v_{1}^{*}\right)=A_{L}^{d} M_{\mathrm{diag}}^{d} A_{R}^{d \dagger}+E_{L}^{d} M_{\mathrm{diag}}^{x d} E_{R}^{d \dagger} .
\end{aligned}
$$


Solving Eq. (38) for $Y^{q}$ and $Y^{q C}$ we get

$$
\begin{aligned}
Y^{q}= & \frac{\sqrt{2}}{\left|v_{1}\right|^{2}-\left|v_{2}\right|^{2}}\left(v_{1}^{*}\left(A_{L}^{u} M_{\mathrm{diag}}^{u} A_{R}^{u \dagger}+E_{L}^{u} M_{\mathrm{diag}}^{x u} E_{R}^{u \dagger}\right)\right. \\
& \left.-v_{2}^{*}\left(A_{L}^{d} M_{\mathrm{diag}}^{d} A_{R}^{d \dagger}+E_{L}^{d} M_{\mathrm{diag}}^{x d} E_{R}^{d \dagger}\right)\right), \\
Y^{q C}= & \frac{\sqrt{2}}{\left|v_{1}\right|^{2}-\left|v_{2}\right|^{2}}\left(-v_{2}\left(A_{L}^{u} M_{\mathrm{diag}}^{u} A_{R}^{u \dagger}+E_{L}^{u} M_{\mathrm{diag}}^{x u} E_{R}^{u \dagger}\right)\right. \\
& \left.+v_{1}\left(A_{L}^{d} M_{\mathrm{diag}}^{d} A_{R}^{d \dagger}+E_{L}^{d} M_{\mathrm{diag}}^{x d} E_{R}^{d^{\dagger}}\right)\right) .
\end{aligned}
$$

From the Yukawa Lagrangian in Eq. (4) the interaction of the neutral components of the bidoublet $\Phi$ with the SM quarks in the gauge basis is given by

$\overline{U_{L}^{0}}\left(Y^{q} \phi_{1}^{0}+Y^{q C} \phi_{2}^{0 *}\right) U_{R}^{0}+\overline{D_{L}^{0}}\left(Y^{q} \phi_{2}^{0}+Y^{q C} \phi_{1}^{0 *}\right) D_{R}^{0}$.

Using Eqs. (39) and (22)-(28) the interactions of the scalars $\phi_{1}^{0}$ and $\phi_{2}^{0}$ with the SM up type quarks in the mass basis can be written as

$$
\begin{aligned}
\overline{U_{L}} A_{L}^{u \dagger}\left(Y^{q} \phi_{1}^{0}+Y^{q C} \phi_{2}^{0 *}\right) A_{R}^{u} U_{R}= & \frac{\sqrt{2}}{v_{-}^{2}} \overline{U_{L}}\left(\left(v_{1}^{*} \phi_{1}^{0}-v_{2} \phi_{2}^{0 *}\right)\left(C_{L}^{u 2} M_{\mathrm{diag}}^{u} C_{R}^{u 2}+C_{L}^{u} S_{L}^{u} M_{\mathrm{diag}}^{x u} S_{R}^{u} C_{R}^{u}\right)\right. \\
& \left.+\left(-v_{2}^{*} \phi_{1}^{0}+v_{1} \phi_{2}^{0 *}\right)\left(V_{L}^{\mathrm{CKM}} M_{\mathrm{diag}}^{d} V_{R}^{\mathrm{CKM} \dagger}+A_{L}^{u \dagger} E_{L}^{d} M_{\mathrm{diag}}^{x d} E_{R}^{d \dagger} A_{R}^{u}\right)\right) U_{R} \\
= & \frac{\sqrt{2}}{v_{-}^{2}} \overline{U_{L}}\left(\phi_{-}^{0} \frac{v_{-}^{2}}{v_{+}}\left(C_{L}^{u 2} M_{\mathrm{diag}}^{u} C_{R}^{u 2}+C_{L}^{u} S_{L}^{u} M_{\mathrm{diag}}^{x u} S_{R}^{u} C_{R}^{u}\right)\right. \\
& +\phi_{+}^{0}\left\{\frac{-2 v_{1}^{*} v_{2}}{v_{+}}\left(C_{L}^{u 2} M_{\mathrm{diag}}^{u} C_{R}^{u 2}+C_{L}^{u} S_{L}^{u} M_{\mathrm{diag}}^{x u} S_{R}^{u} C_{R}^{u}\right)\right. \\
& \left.\left.+v_{+}\left(V_{L}^{\mathrm{CKM}} M_{\mathrm{diag}}^{d} V_{R}^{\mathrm{CKM} \dagger}+V_{L}^{\mathrm{CKM}} C_{L}^{d-1} S_{L}^{d} M_{\mathrm{diag}}^{x d} S_{R}^{d} C_{R}^{d-1} V_{R}^{\mathrm{CKM} \dagger}\right)\right\}\right) U_{R},
\end{aligned}
$$

where the two orthogonal fields $\phi_{-}^{0}$ and $\phi_{+}^{0}$ are given by [52]

$$
\phi_{+}^{0}=\frac{1}{v_{+}}\left(-v_{2}^{*} \phi_{1}^{0}+v_{1} \phi_{2}^{0 *}\right), \quad \phi_{-}^{0}=\frac{1}{v_{+}}\left(v_{1}^{*} \phi_{1}^{0}+v_{2} \phi_{2}^{0 *}\right) .
$$

And $v_{ \pm}^{2}=\left|v_{1}\right|^{2} \pm\left|v_{2}\right|^{2}$. Similarly the interaction terms for the SM down type quarks in the mass basis is given by

$$
\begin{aligned}
\overline{D_{L}} A_{L}^{d \dagger}\left(Y^{q} \phi_{2}^{0}+Y^{q C} \phi_{1}^{0 *}\right) A_{R}^{d} D_{R}= & \frac{\sqrt{2}}{v_{-}^{2}} \overline{D_{L}}\left(\phi_{-}^{0 *} \frac{v_{-}^{2}}{v_{+}}\left(C_{L}^{d 2} M_{\mathrm{diag}}^{d} C_{R}^{d 2}+C_{L}^{d} S_{L}^{d} M_{\mathrm{diag}}^{x d} S_{R}^{d} C_{R}^{d}\right)\right. \\
& +\phi_{+}^{0 *}\left\{\frac{-2 v_{1} v_{2}^{*}}{v_{+}}\left(C_{L}^{d 2} M_{\mathrm{diag}}^{d} C_{R}^{d 2}+C_{L}^{d} S_{L}^{d} M_{\mathrm{diag}}^{x d} S_{R}^{d} C_{R}^{d}\right)\right. \\
& \left.\left.+v_{+}\left(V_{L}^{\mathrm{CKM} \dagger} M_{\mathrm{diag}}^{u} V_{R}^{\mathrm{CKM}}+V_{L}^{\mathrm{CKM} \dagger} C_{L}^{u-1} S_{L}^{u} M_{\mathrm{diag}}^{x u} S_{R}^{u} C_{R}^{u-1} V_{R}^{\mathrm{CKM}}\right)\right\}\right) D_{R} .
\end{aligned}
$$

From Eqs. (41) and (43) it can be concluded that the interactions $\phi_{-}^{0}$ is flavor-diagonal but the interactions of $\phi_{+}^{0}$ is flavor-changing. $\phi_{+}^{0}$ interactions are flavor-changing because the matrix $V_{L}^{\mathrm{CKM}}$ which is the measured CKM matrix is not diagonal. Although the matrix $V_{R}^{\mathrm{CKM}}$ can be nondiagonal there is no experimental constraint which forces it to be non-diagonal and hence $V_{R}^{\mathrm{CKM}}$ can be taken to be diagonal by proper choice of Yukawa couplings. In left-right symmetric model the field $\phi_{-}^{0}$ is always flavorconserving in nature [52]. But in the 221 model we are discussing $\phi_{-}^{0}$ can also have flavor-violating interactions for general mixing patterns between VLQs and the SM quarks. It is the form of the mixing matrices in Eqs. (24) and(27) which ensures that $\phi_{-}^{0}$ have flavor-conserving interactions.

In general $\phi_{+}^{0}$ is not a mass eigenstate and when both $v_{1}$ and $v_{2}$ are nonzero all the neutral mass eigenstates will contain $\phi_{+}^{0}$. And hence all of the neutral scalars have to be heavy to avoid constraints from FCNC interactions. Therefore when both $v_{1}$ and $v_{2}$ take nonzero values it will be impossible to get a light mass eigenstate at $125 \mathrm{GeV}$ which do not have flavor violating interactions. This scenario has been extensively discussed in the context of left-right symmetric model in [53].

Hence to have a flavor-conserving Higgs at $125 \mathrm{GeV}$ we made the choice $v_{2}=0$, which gives 


$$
v_{+}=v_{-}=v_{1}, \quad \phi_{+}^{0}=\phi_{2}^{0 *}, \quad \phi_{-}^{0}=\phi_{1}^{0} .
$$

The VEV $v_{1}$ has been considered as a real parameter. As we will show it is possible to choose the parameters of the scalar potential in the model such that $\phi_{2}^{0}$ do not mix with any other scalars and can be made heavy to avoid large neutral flavor-changing interactions. $\phi_{1}^{0}$ will be a part of the observed $125 \mathrm{GeV}$ Higgs. For $v_{2}=0$ the interactions of flavor conserving Higgs $\phi_{1}^{0}$ with the SM type quarks are given by

$$
\begin{aligned}
& \frac{\sqrt{2}}{v_{1}} \overline{U_{L}}\left(\phi_{1}^{0}\left(C_{L}^{u 2} M_{\mathrm{diag}}^{u} C_{R}^{u 2}+C_{L}^{u} S_{L}^{u} M_{\mathrm{diag}}^{x u} S_{R}^{u} C_{R}^{u}\right)\right) U_{R}+\text { H.c. } \\
& \frac{\sqrt{2}}{v_{1}} \overline{D_{L}}\left(\phi_{1}^{0 *}\left(C_{L}^{d 2} M_{\mathrm{diag}}^{d} C_{R}^{d 2}+C_{L}^{d} S_{L}^{d} M_{\mathrm{diag}}^{x d} S_{R}^{d} C_{R}^{d}\right)\right) D_{R}+\text { H.c. }
\end{aligned}
$$

And the interactions for the flavor-violating Higgs $\phi_{2}^{0}$ are given by

$$
\begin{aligned}
& \frac{\sqrt{2}}{v_{1}} \overline{U_{L}}\left(\phi_{2}^{0 *}\left\{\left(V_{L}^{\mathrm{CKM}} M_{\mathrm{diag}}^{d} V_{R}^{\mathrm{CKM} \dagger}+A_{L}^{u \dagger} E_{L}^{d} M_{\mathrm{diag}}^{x d} E_{R}^{d^{\dagger} \dagger} A_{R}^{u}\right)\right\}\right) U_{R} \\
& + \text { H.c., } \\
& \frac{\sqrt{2}}{v_{1}} \overline{D_{L}}\left(\phi_{2}^{0}\left\{\left(V_{L}^{\mathrm{CKM} \dagger} M_{\mathrm{diag}}^{u} V_{R}^{\mathrm{CKM}}+A_{L}^{d \dagger} E_{L}^{u} M_{\mathrm{diag}}^{x u} E_{R}^{u \dagger} A_{R}^{d}\right)\right\}\right) D_{R} \\
& \quad+\text { H.c. }
\end{aligned}
$$

To study the nature of interactions of the neutral components from the doublets $H_{1}$ and $H_{2}$ we list the Yukawa couplings in terms of the mixing matrices as below [for mixing matrices of the form taken in Eq. (24) and $\left.v_{2}=0\right]$

$$
\begin{aligned}
Y^{q} \frac{v_{1}}{\sqrt{2}} & =\widehat{A_{L}^{u}}\left(C_{L}^{u} M_{\mathrm{diag}}^{u} C_{R}^{u}+S_{L}^{u} M_{\mathrm{diag}}^{x u} S_{R}^{u}\right) \widehat{A}_{R}^{\dagger}, \\
Y^{q x u} \frac{v_{3}}{\sqrt{2}} & =\widehat{A_{L}^{u}}\left(C_{L}^{u} M_{\mathrm{diag}}^{u} S_{R}^{u}-S_{L}^{u} M_{\mathrm{diag}}^{x u} C_{R}^{u}\right), \\
\mu & =\left(S_{L}^{u} M_{\mathrm{diag}}^{u} C_{R}^{u}-C_{L}^{u} M_{\mathrm{diag}}^{x u} S_{R}^{u}\right) \widehat{A}_{R}^{\dagger}, \\
Y^{x q x u} \frac{u}{\sqrt{2}} & =S_{L}^{u} M_{\mathrm{diag}}^{u} S_{R}^{u}+C_{L}^{u} M_{\mathrm{diag}}^{x u} C_{R}^{u} .
\end{aligned}
$$

Similar relations for the matrices $Y^{q C}, Y^{q x d}, \mu$ and $Y^{x q x d}$ can be found by using the mixing matrices for the down-type quark sector. Note that the mixing angles and the mass eigenvalues should be such that both the up-quark sector and the down-quark sector yield the same $\mu$ matrix.

The fields $\chi^{0}$ and $\chi^{\prime 0}$ in Eq. (7) are the neutral components of the doublets $H_{1}$ and $H_{2}$, respectively. The interactions of the up-type SM mass eigenstate quarks with $\chi^{0}$ and $\chi^{\prime 0}$ can be derived from the terms proportional to $Y_{i j}^{q x u}$ and $Y_{i j}^{x q x u}$, respectively in the Yukawa Lagrangian. These interactions are given by

$$
\begin{aligned}
Y_{i j}^{q x u} \overline{Q_{i L}} x u_{j R}^{0} H_{1} & \supset \chi^{0} \overline{U_{L}^{0}} Y^{q x u} X U_{R}^{0} \\
& \supset \frac{\sqrt{2}}{v_{3}} \chi^{0} \overline{U_{L}}\left[C _ { L } ^ { u } \left(C_{L}^{u} M_{\mathrm{diag}}^{u} S_{R}^{u}\right.\right. \\
& \left.\left.-S_{L}^{u} M_{\mathrm{diag}}^{x u} C_{R}^{u}\right) S_{R}^{u}\right] U_{R} \\
\text { and } \quad Y_{i j}^{x q x u} \overline{X Q_{i L}} x u_{j R}^{0} H_{2} \supset & \chi^{\prime 0} \overline{X U_{L}^{0}} Y^{x q x u} X U_{R}^{0} \\
\supset & \frac{\sqrt{2}}{u} \chi^{\prime 0} \overline{U_{L}}\left[S _ { L } ^ { u } \left(S_{L}^{u} M_{\mathrm{diag}}^{u} S_{R}^{u}\right.\right. \\
& \left.\left.+C_{L}^{u} M_{\mathrm{diag}}^{x u} C_{R}^{u}\right) S_{R}^{u}\right] U_{R} .
\end{aligned}
$$

The superset sign $(\supset)$ has been used in the above equations to highlight the terms containing only the fields $\chi^{0}, \chi^{\prime 0}$ and the SM up-type mass eigenstate quarks $\left(U_{L}, U_{R}\right)$. From Eq. (48) it can be concluded that the scalars $\chi^{0}$ and $\chi^{10}$ do not have flavor-changing interactions with the SM mass eigenstate quarks.

Based on the above discussions, we denote the field $\phi_{2}^{0}$ as the FCNH (flavor-changing neutral Higgs) scalar and the fields $\phi_{1}^{0}, \chi^{0}$ and $\chi^{\prime 0}$ as the three non-FCNH scalars in the model. One linear combination of the three non-FCNH scalars will be the $125 \mathrm{GeV}$ SM Higgs and the other linear combinations can lie at the sub-TeV scale.

The special cases for the matrices $Y^{q x u}$ and $\mu$ which will play an important role in the phenomenology of the VLQs are given by:

$$
Y^{q x u}=0 \Rightarrow \tan \theta_{L}^{i}=\frac{m_{i}}{m_{x u_{i}}} \tan \theta_{R}^{i}
$$

and

$$
\mu=0 \Rightarrow \tan \theta_{R}^{i}=\frac{m_{i}}{m_{x u_{i}}} \tan \theta_{L}^{i},
$$

where $i \in(u, c, t)$.

For simplicity we consider the scenario where the three matrices $\widehat{A_{L}^{d}}, \widehat{A_{R}^{u}}$ and $\widehat{A_{R}^{d}}$ are equal to $\mathbb{1}$. The choice for the Yukawa couplings which will lead to such a scenario can be made by using $\widehat{A_{L}^{d}}=\mathbb{1}, \widehat{A_{R}^{u}}=\mathbb{1}$ and $\widehat{A_{R}^{d}}=\mathbb{1}$ in Eq. (47). From Eq. (47) it can be observed that the complete determination of Yukawa couplings will depend on the SM quark masses, desired values of VLQ masses, desired values of mixing angles, VEVs and on the form of the matrix $\widehat{A_{L}^{u}}$. The measured CKM matrix will enter through the matrix $\widehat{A_{L}^{u}}$ because, $V_{L}^{\mathrm{CKM}}=A_{L}^{u \dagger} A_{L}^{d}=C_{L}^{u} \widehat{A_{L}^{u} \dagger} \widehat{A_{L}^{d}} C_{L}^{d}$.

\section{PHENOMENOLOGICAL ASPECTS}

So far we have discussed about the methodology to get rid of any tree level $Z$ boson FCNC and to achieve a $125 \mathrm{GeV}$ Higgs with no flavor-changing interactions. Now we shall discuss some of the phenomenological implications of the model. To do that we will choose a 
representative mass spectrum for the exotic particles in our model and then discuss the possible collider signals which can be explored at the LHC.

\section{A. Scalar and gauge boson mass spectrum}

To relate the gauge eigenstates with the mass eigenstates for the scalar sector, we introduce three $4 \times 4$ matrices $Z^{E}$, $Z^{O}$ and $Z^{C}$ for $C P$ even sector, $C P$ odd sector and charged sector, respectively. The relations are given by

$$
\begin{aligned}
\left(\begin{array}{l}
h_{1} \\
h_{2} \\
h_{3} \\
h_{4}
\end{array}\right) & =Z^{E}\left(\begin{array}{c}
\phi_{1}^{0 r} \\
\phi_{2}^{0 r} \\
\chi^{0 r} \\
\chi^{0 r}
\end{array}\right), \quad\left(\begin{array}{c}
G_{1} \\
G_{2} \\
A_{1} \\
A_{2}
\end{array}\right)=Z^{O}\left(\begin{array}{c}
\phi_{1}^{0 i} \\
\phi_{2}^{0 i} \\
\chi^{0 i} \\
\chi^{0 i}
\end{array}\right), \\
\left(\begin{array}{c}
G_{1}^{+} \\
G_{2}^{+} \\
h_{1}^{+} \\
h_{2}^{+}
\end{array}\right) & =Z^{C}\left(\begin{array}{c}
\phi_{1}^{+} \\
\phi_{2}^{+} \\
\chi^{\prime+} \\
\chi^{+}
\end{array}\right) .
\end{aligned}
$$

$$
\begin{aligned}
Z^{E} & =\left(\begin{array}{cccc}
0.999 & 0 & \sim 10^{-8} & 0.034 \\
-0.034 & 0 & \sim 10^{-7} & 0.999 \\
0 & 1 & 0 & 0 \\
\sim-10^{-8} & 0 & \sim 1 & \sim-10^{-7}
\end{array}\right), \\
Z^{C} & =\left(\begin{array}{cccc}
0.999 & 0 & 0 & 0.028 \\
0 & 0.02 & 0.999 & 0 \\
-0.028 & 0 & 0 & 0.999 \\
0 & 0.999 & -0.02 & 0
\end{array}\right) .
\end{aligned}
$$

The mass eigenvalues are: $m_{h_{1}} \approx 125 \mathrm{GeV}, m_{h_{2}} \approx$ $300 \mathrm{GeV}, m_{h_{3}} \approx 8.5 \mathrm{TeV}, m_{h_{4}} \approx 17 \mathrm{TeV}, m_{A_{1}} \simeq 300 \mathrm{GeV}$, $m_{A_{2}} \simeq 8.5 \mathrm{TeV}, m_{h_{1}^{+}} \simeq 363 \mathrm{GeV}$ and $m_{h_{2}^{+}} \simeq 8.5 \mathrm{TeV}$. We shall keep this spectrum for the scalars as our choice for the collider analysis presented later.

The mixing matrices clearly show that the neutral scalars $h_{3}$ and $A_{2}$, which are basically $\phi_{2}^{0 r}$ and $\phi_{2}^{0 i}$, respectively, have been kept unmixed with other neutral scalars, because both $\phi_{2}^{0 r}$ and $\phi_{2}^{0 i}$ have FCNH interactions as discussed earlier.

For the choice $u=12 \mathrm{TeV}$ and for $g_{2}=g_{1}$, the mass values for $W^{\prime}$ and $Z^{\prime}$ are $M_{W^{\prime}} \simeq 4 \mathrm{TeV}$ and $M_{Z^{\prime}} \simeq 4.7 \mathrm{TeV}$ respectively, and both of them satisfies the current lower bounds obtained by the ATLAS collaboration $[54,55]$. Also the $Z-Z^{\prime}$ mixing angle is small $\left(\theta_{z z^{\prime}} \simeq 10^{-4}\right)$ and satisfies the constraint from the electroweak precision data [56]. Note that for $v_{2}=0$, the $W-W^{\prime}$ mixing angle $\theta_{\mathrm{ww}^{\prime}}$ [see Eq. (14)] is zero.
Here $h_{1}, h_{2}, h_{3}, h_{4}$ are $C P$ even scalar mass eigenstates, $A_{1}$ and $A_{2}$ are $C P$ odd scalar mass eigenstates and $h_{1}^{+}, h_{2}^{+}$are charged scalar mass eigenstates. $G_{1}, G_{2}$ are neutral Goldstone bosons and $G_{1}^{+}, G_{2}^{+}$are the charged Goldstone bosons. As discussed in the previous section, the field $\phi_{2}{ }^{0}$ will have FCNH interactions while the fields $\phi_{1}{ }^{0}, \chi^{0}$ and $\chi^{\prime 0}$ have no FCNH interactions. Therefore, any mass eigenstate formed out of the linear combinations of the three fields $\phi_{1}{ }^{0}, \chi^{0}$ and $\chi^{\prime 0}$ can lie at sub-TeV scale. A typical example of the composition of the sub-TeV scalar states can be arranged through the following choice of the parameter values in our model:

$$
\begin{aligned}
& \left\{M_{2}, \alpha_{1}, \alpha_{2}, \beta_{1}, \beta_{2}, \lambda_{2}, \lambda_{3}, \lambda_{4}, \rho_{3}\right\}=0, \\
& u=12 \mathrm{TeV}, \quad M_{1}=-0.3 \mathrm{GeV}, \\
& v_{2}=0, \quad v_{3}=7 \mathrm{GeV}, \\
& \rho_{1}=0.1, \quad \rho_{2}=1, \quad \lambda_{1}=0.13, \\
& \beta_{3}=1.4 \quad \text { and } \quad \alpha_{3}=1 .
\end{aligned}
$$

For the above choice of parameters the mixing matrices are given by

$$
Z^{O}=\left(\begin{array}{cccc}
0.999 & 0 & 0.02 & 0.028 \\
-0.02 & 0 & 0.999 & 0 \\
-0.028 & 0 & \sim-10^{-4} & 0.999 \\
0 & 1 & 0 & 0
\end{array}\right)
$$

\section{B. Production and decay of the vectorlike quarks}

As color triplets, the VLQs will be pair-produced at the LHC mostly through strong interactions. The pair production cross section at LHC with different center-of-mass energies and as a function of the mass of $x u_{3}$ has been shown in Fig. 1. We have used NN23LO1 [57] parton distribution function with default factorization and renormalization scale in MADGRAPH5_AMC@NLO for our estimates.

For a given set of allowed values for the parameters in the model, the six VLQs (three up-type and three downtype) will mostly have different signatures depending on the generation they belong to. To discuss the phenomenology and for simplicity we consider the third generation uptype VLQ $x u_{3}$ to be the lightest one among all VLQs in the model. Since we have already discussed in detail the mixing between VLQs and SM quarks, we list the relevant interaction terms for $x u_{3}$ in Table II. As the interactions 


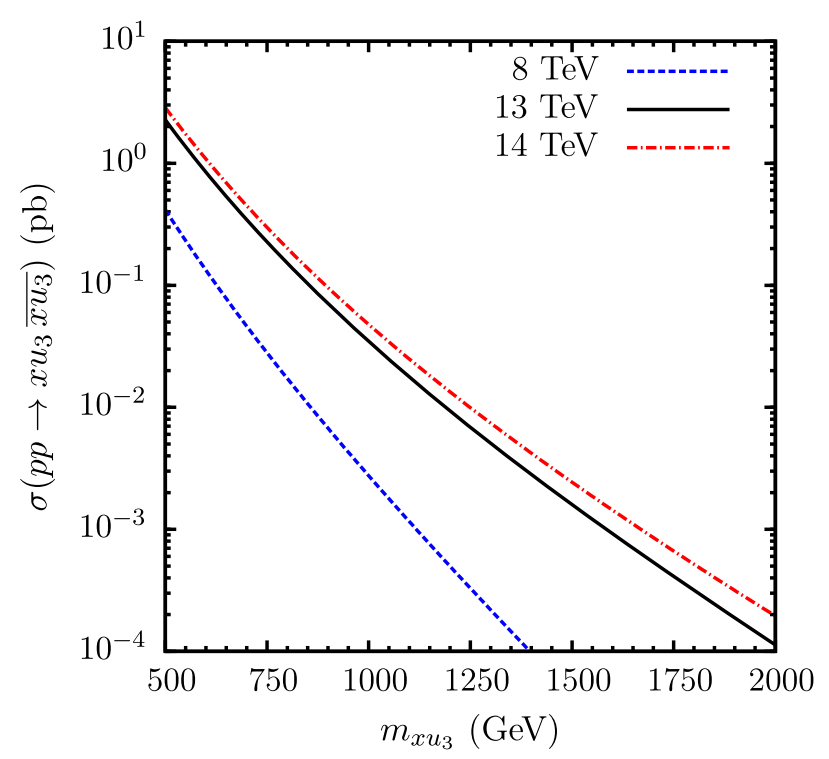

FIG. 1. Production cross section for the VLQ $x u_{3}$ for different masses for $8 \mathrm{TeV}, 13 \mathrm{TeV}$ and $14 \mathrm{TeV}$ LHC.

with the physical scalar fields would look quite cumbersome and messy, we have chosen to show the interaction of the physical (mass eigenstates) fermions with the scalars in the gauge eigenbasis. The interaction terms with the physical scalars can be obtained by using the rotated fields in terms of the elements of $4 \times 4$ matrices $Z^{E}, Z^{O}$ and $Z^{C}$. For example, the interaction term for $A_{1}$ will be

$$
\begin{aligned}
A_{1} \bar{t} x u_{3}: & {\left[\left(Z^{O}\right)_{13}^{T} K_{\phi_{1}^{0 i}}\left(C_{\phi_{1}^{0 i}}^{S}+C_{\phi_{1}^{0 i}}^{P} \gamma^{5}\right)\right.} \\
& +\left(Z^{O}\right)_{23}^{T} K_{\phi_{2}^{0 i}}\left(C_{\phi_{2}^{0 i}}^{S}+C_{\phi_{2}^{0 i}}^{P} \gamma^{5}\right) \\
& +\left(Z^{O}\right)_{33}^{T} K_{\chi^{10 i}}\left(C_{\chi^{0 i}}^{S}+C_{\chi^{0 i}}^{P} \gamma^{5}\right) \\
& \left.+\left(Z^{O}\right)_{43}^{T} K_{\chi^{0 i}}\left(C_{\chi^{0 i}}^{S}+C_{\chi^{0 i}}^{P} \gamma^{5}\right)\right] .
\end{aligned}
$$

The possible final states that $x u_{3}$ can decay to are $t Z, t h_{1}$, $b W^{+}, t h_{2}, t A_{1}$ and $b h_{1}^{+}$, since the scalars $h_{3}, h_{4}, A_{2}, h_{2}^{+}$and the new gauge bosons $Z^{\prime}, W^{\prime}$ are heavier compared to the VLQ $x u_{3}$. For small mixing angles the "nonstandard" decay modes $\left(t h_{2}, t A_{1}, b h_{1}^{+}\right)$will mostly dominate over the standard decay modes $\left(t Z, t h_{1}, b W^{+}\right)$because of the presence of direct Yukawa interaction term, $Y^{q x u}$ in the Lagrangian. The standard decay modes will start dominating once $Y^{q x u}$ tends to zero. This feature is illustrated in Fig. 2 which shows the branching ratios for different decay modes for a $800 \mathrm{GeV} x u_{3}$ as a function of mixing angles, where we have fixed $\sin \theta_{R}^{t}=10^{-3}$ and varied $\sin \theta_{L}^{t}$ accordingly.

We have considered small mixing angles $\left(\simeq 10^{-3}\right)$ to avoid constraints from flavor sector and electroweak precision data [16,38-42] and as an example, we have checked that the contribution of VLQs to the $K-\bar{K}$ oscillation parameter $\Delta m_{K}$ is few orders of magnitude less compared to the SM value. We find that the nonstandard decay modes dominate the standard decay modes except where $\frac{\sin \theta_{L}^{t}}{\sin \theta_{R}^{t}}$ lies in the small range $0.2-0.23$. We can understand this feature of the decay probability by looking at the interaction terms $\chi^{0 r} \bar{t} x u_{3}, \chi^{0 i} \bar{t} x u_{3}$ and $\chi^{-} \bar{b} x u_{3}$ from Table II. In the limit $\frac{\tan \theta_{L}^{t}}{\tan \theta_{R}^{t}}=\frac{m_{t}}{m_{x u_{3}}}$, the coupling strengths for the interactions $\chi^{0 r} \bar{t} x u_{3}$ and $\chi^{0 i} \bar{t} x u_{3}$ is identically zero. Moreover, the same limit along with small values of $\sin \theta_{L}^{t}$ and $\sin \theta_{R}^{b}$ make the coupling strength for the interaction $\chi^{-} \bar{b} x u_{3}$ negligibly small. Thus when the ratio of mixing angles become

$$
\frac{\sin \theta_{L}^{t}}{\sin \theta_{R}^{t}} \simeq \frac{\tan \theta_{L}^{t}}{\tan \theta_{R}^{t}}=\frac{m_{t}}{m_{x u_{3}}} \simeq \frac{173}{800} \simeq 0.216,
$$

the interactions with the scalars $h_{2}, A_{1}$ and $h_{1}^{+}$goes to zero. Consequently the decays of $x u_{3}$ to the SM particles enhances. In the next section we study the possible collider signatures for the scenario where the branching ratios for the VLQ $x u_{3}$ lie away from the standard mode dominated region such that after production $x u_{3}$ decays to one of the final states from $t h_{2}, t A_{1}, b h_{1}^{+}$.

The collider signatures of $x u_{3}$ will eventually depend on the decay modes of the scalars $h_{2}, A_{1}$ and $h_{1}^{+}$. From Eq. (51) and Eq. (52) we note that the scalar $h_{2}$ is made up of a very small component $\left(\sim 10^{-2}\right)$ of one of the real neutral part of the bidoublet Higgs field $\left(\phi_{1}^{0 r}\right)$ and a large component of the real neutral part of $H_{1}$, i.e., $\chi^{0 r}$. From the Yukawa terms in the Lagrangian it can be seen that $H_{1}$ gives mass to the charged leptons but there is no Yukawa interaction term involving SM quarks and $H_{1}$. Hence the strength of the Yukawa interaction for the mass eigenstate $h_{2}$ with the SM quarks is negligible compared to the coupling strength with the leptons. Hence $h_{2}$ will mostly decay to leptons compared to the SM quarks. The same argument is also applicable for $A_{1}$ and $h_{1}^{+}$, because all of them are largely composed of $H_{1}$. Note that the charged leptons get masses from the VEV of the $S U(2)_{L}$ doublet scalar $H_{1}$ which gets a small VEV, $\frac{v_{3}}{\sqrt{2}} \sim 5 \mathrm{GeV}$. The Yukawa coupling strengths for the scalars $h_{2}, A_{1}, h_{1}^{+}$with the leptons from different generations follow the mass hierarchy and with more than $99 \%$ probability the scalar $h_{2}$ and $A_{1}$ will decay to $\tau^{+} \tau^{-}$whereas $h_{1}^{+}$will decay to $\tau^{+} \nu_{\tau}$.

\section{Benchmark points}

For the collider analysis we have chosen three benchmark points based on $x u_{3}$ mass. The pair production cross section and the branching ratios for different benchmarks are given in Table III. For all the three cases the masses for the scalars $h_{2}, A_{1}$ and $h_{1}^{+}$are kept fixed at $300 \mathrm{GeV}$, $300 \mathrm{GeV}$ and $363 \mathrm{GeV}$, respectively. Due to the smallness of the Yukawa couplings with the SM quarks, the scalars $h_{2}$ 
TABLE II. The interaction terms including scalars are of the form $\phi K_{\phi}\left(C_{\phi}^{S}+C_{\phi}^{P} \gamma^{5}\right) x u_{3}$ and including gauge bosons are of the form $a_{\mu} \bar{t} K_{a} \gamma^{\mu}\left(C_{a}^{V}+C_{a}^{A} \gamma^{5}\right) x u_{3}$. The interactions for the physical scalars can be obtained using the transformations given by Eq. (51).

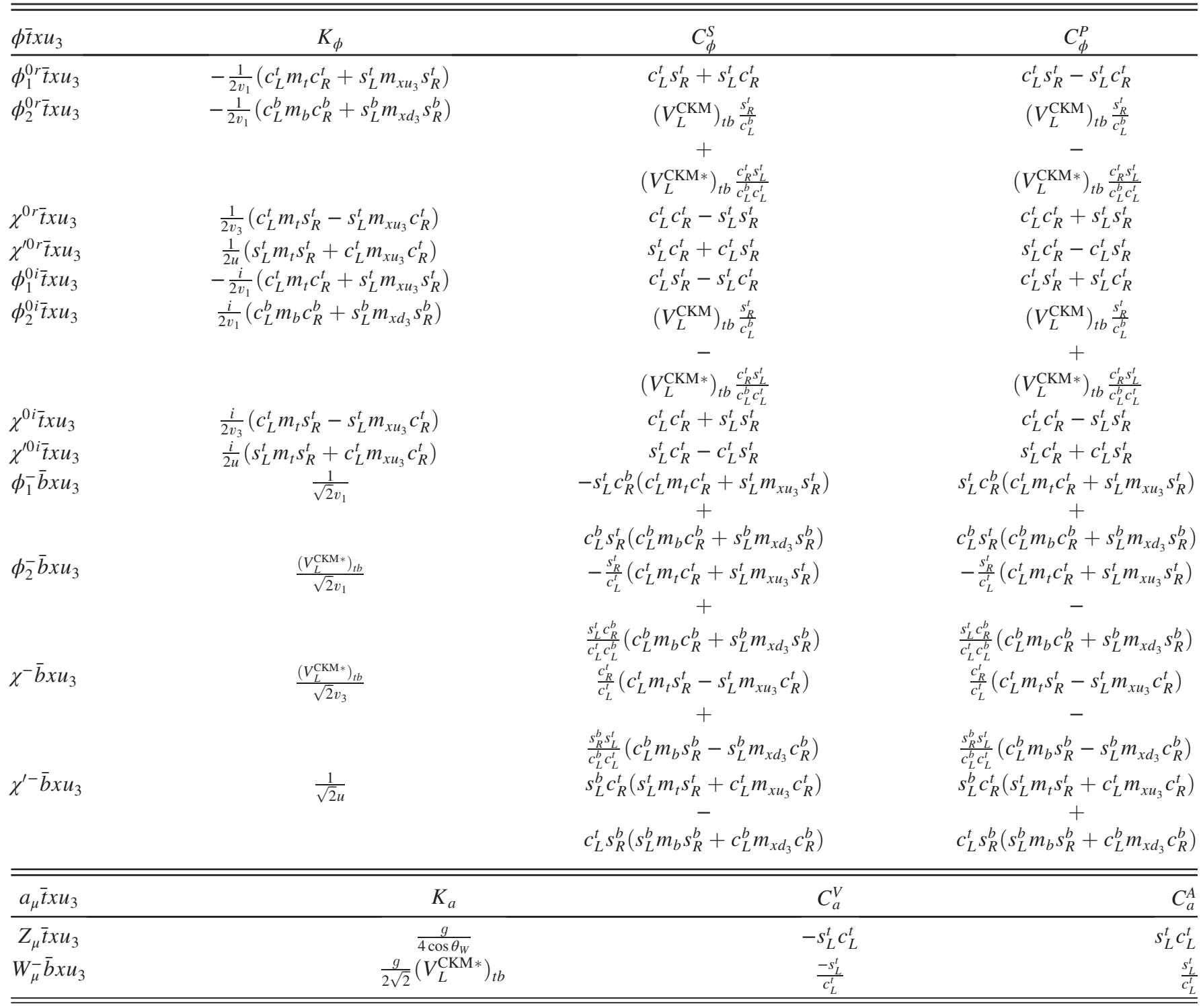

and $A_{2}$ cannot be produced efficiently at the LHC via gluon fusion and the production cross sections of them are few tens of $\mathrm{fb}$ for $13 \mathrm{TeV}$ center of mass energy. Hence, the experimental limits on on their masses are fairly weak. Note that the Yukawa couplings of these scalars with the VLQs are also very small and the VLQ loops contribute very less toward their production. The scenario is almost same as the lepton-specific two-Higgs doublet model where the limit on the massive states are of the order of 180 $200 \mathrm{GeV}$ [58]. Since the production cross-section falls off rapidly for higher masses we have used an integrated luminosity of $100 \mathrm{fb}^{-1}$ for $\mathrm{BP} 1$ whereas $3000 \mathrm{fb}^{-1}$ luminosity is used for the analysis of BP2 and BP3.

We would like to emphasize that the benchmark points chosen here are fairly general as long as the mass of $x u_{3}$ is greater than the masses of the scalars $h_{2}, A_{1}$ and $h_{1}^{+}$, such that the nonstandard decay modes are kinematically allowed. From Fig. 2 it can be observed that the decay branchings depend mildly on the ratio of the mixing angles $\theta_{L}$ and $\theta_{R}$, once we are away from the narrow peak region. Also the decay branchings of $h_{2}, A_{1}$ and $h_{1}^{+}$to tau leptons depend on the Yukawa couplings and are almost independent of the masses of the scalars.

\section{COLLIDER ANALYSIS}

Now we consider the collider signatures of $x u_{3}$ for $13 \mathrm{TeV}$ LHC in the scenario where the pair produced $x u_{3}$ will decay to the final states $t h_{2}, t A_{1}, b h_{1}^{+}$and the scalars $h_{2}, A_{1}$ and $h_{1}^{+}$will further decay to tau leptons. Taking into 


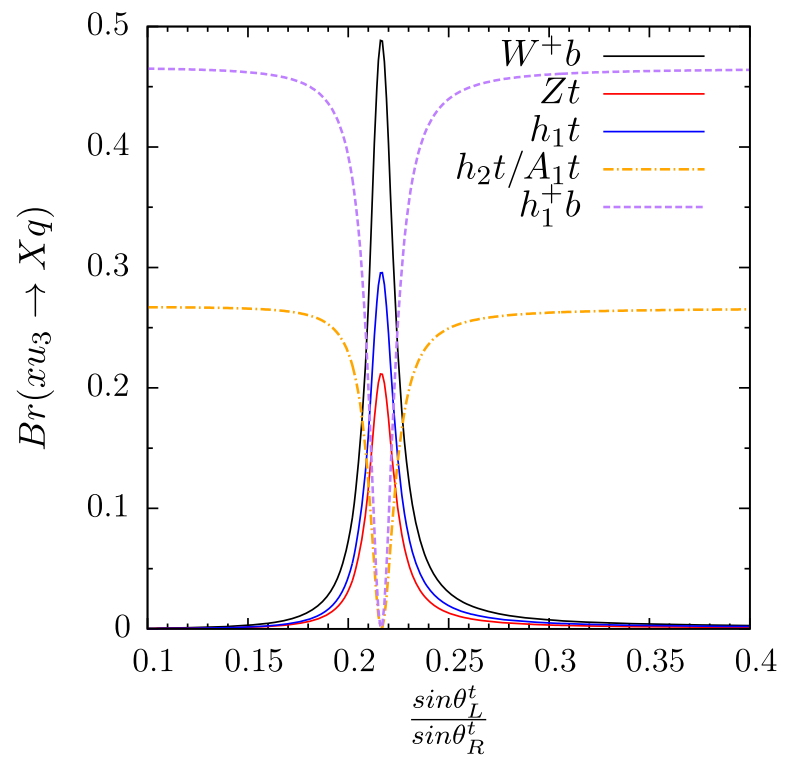

FIG. 2. Branching ratios for different decay modes of the VLQ $x u_{3}$ as a function of mixing angle $\theta_{L}^{t}$. For the plot $m_{x u_{3}}=800 \mathrm{GeV}, \quad m_{h_{2}}=m_{A_{1}}=300 \mathrm{GeV}, \quad m_{h_{1}^{+}}=363 \mathrm{GeV}$, $m_{x d_{3}}=5 \mathrm{TeV}, \sin \theta_{L}^{b}=10^{-4}, \sin \theta_{R}^{b} \simeq 10^{-4}$ and $\sin \theta_{R}^{t}=10^{-3}$.

account all possible decay chains of $x u_{3}$, Fig. 3 shows all possible final states that can arise from the pair production of $x u_{3}$. Since each final state contains at least two tau leptons and at least one $b$ quark, we choose the final state with at least two $\tau$-tagged jets, at least three non $\tau$-tagged jets among which at least one is $b$-tagged, and at least one lepton $(\geq 3 j(1 b)+\geq 2 \tau+\geq 1 l)$ for the collider analysis.

The possible SM processes that can contribute as background to the above choice of final state are the following:

(i) $p p \rightarrow t \bar{t}+$ jets,

(ii) $p p \rightarrow t \bar{t} l^{+} l^{-}+$jets and $p p \rightarrow t \bar{t} \tau^{+} \tau^{-}+$jets,

(iii) $p p \rightarrow t \bar{t} l^{+} \nu_{l}+$ jets and $p p \rightarrow t \bar{t} \tau^{+} \nu_{\tau}+$ jets,

(iv) $p p \rightarrow Z Z Z$,

(v) $p p \rightarrow W^{ \pm} / Z+$ jets.

Among all the above possible backgrounds the most dominant one is $t \bar{t}+$ jets. Although the cross section for the backgrounds $W^{ \pm} / Z+$ jets is large it is possible to get rid of this by a large $E T$ requirement which we have used in our analysis. The contribution of $Z Z Z$ will be negligible because of its small cross section. Hence we consider only the first three of the above list of backgrounds for the collider analysis in the context of $13 \mathrm{TeV}$ LHC.

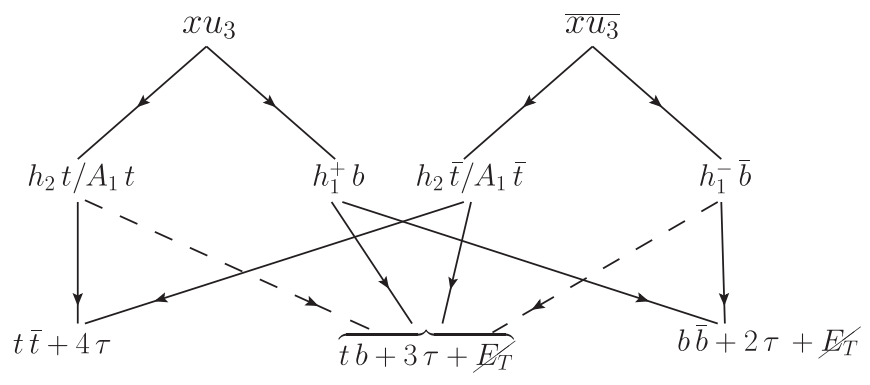

FIG. 3. All possible final states resulting from the pair production of $x u_{3}$ and their subsequent nonstandard decay.

To study the collider phenomenology we implemented the model in the spectrum-generator-generator SARAH [59]. The source code generated by SARAH for the spectrum generator SPHENO [60] has been used in SPHENO to study the spectrum of the model. The files generated by SARAH in the UFO format and the spectrum file generated by SPHENO has been used in MADGRAPH 5 [61] for event generation for the signal. The background events have also been generated using MADGRAPH 5. For showering and hadronization we used PythIA 6 [62] interfaced in MADGRAPH 5. DELPHES 3 [63] within CMS environment has been used to take into account the detector effects and also for reconstruction of the final state objects. The anti- $k_{T}$ algorithm with cone size 0.5 have been used for the jet reconstruction. For the reconstruction of the jets, FASTJET [64] embedded in DELPHES has been used. MADANALYSIS 5 [65] package has been used for the event-analysis using the event format ROOT and LHCO.

The selection criteria for the final state objects in the reconstructed events are such that a non $\tau$-tagged jet with $p_{T}(j)>20 \mathrm{GeV}$ and $|\eta(j)|<2.5$ is considered in the event, an electron or a muon with $p_{T}(l)>10 \mathrm{GeV}$ and $|\eta(l)|<2.5$ are considered in the event, a $\tau$-tagged jet with $p_{T}(\tau)>20 \mathrm{GeV}$ and $|\eta(\tau)|<2.5$ is considered in the event. Note that here $j$ denotes a non $\tau$-tagged jet, $\tau$ denotes a $\tau$-tagged jet. A non $\tau$-tagged jet $(j)$ is either a light jet or a $b$-tagged jet. The minimum angular separation between all final state objects satisfy $\Delta R>0.4$. The $\tau$ tagging and mistagging efficiencies are incorporated in DELPHES3 as reported by the ATLAS collaboration [66]. We operate our simulation on the Medium tag point for which the tagging efficiency of 1-prong (3-prong) $\tau$ decay is $70 \%(60 \%)$ and the corresponding mistagging rate is $1 \%(2 \%)$.

TABLE III. Different benchmark scenarios we have used in collider analysis.

\begin{tabular}{lccccc}
\hline \hline Benchmarks & $m_{x u_{3}}$ & $\operatorname{Br}\left(x u_{3} \rightarrow h_{2} t\right)$ & $\operatorname{Br}\left(x u_{3} \rightarrow A_{1} t\right)$ & $\operatorname{Br}\left(x u_{3} \rightarrow h_{1}^{+} b\right)$ & $\sigma\left(p p \rightarrow x u_{3}, \overline{x u_{3}}\right)$ \\
\hline BP1 & $1 \mathrm{TeV}$ & 0.26 & 0.26 & 0.48 & $32.33 \mathrm{fb}$ \\
BP2 & $1.5 \mathrm{TeV}$ & 0.255 & 0.255 & 0.49 & $1.554 \mathrm{fb}$ \\
BP3 & $2 \mathrm{TeV}$ & 0.25 & 0.25 & 0.5 & $0.113 \mathrm{fb}$ \\
\hline \hline
\end{tabular}




\section{A. BP1: $m_{x u_{3}}=1 \mathrm{TeV}$ with $100 \mathrm{fb}^{-1}$}

For BP1 the production cross section and different branching ratios are tabulated in Table III. For the background simulation we generated $p p \rightarrow t \bar{t}$ events up to two additional jets at the leading order accuracy and used shower- $K_{T}$ matching scheme in MADGRAPH 5 to avoid the double counting between the partonic events and showered events. For the event analysis we used the cross section for $13 \mathrm{TeV}$ at the NNLO accuracy for top quark pair production, i.e., $815.96 \mathrm{pb}$ [67]. By following the same procedure we have generated both the $t \bar{t} l^{+} l^{-}$and $t \bar{t} l \nu_{l}$ events up to two additional jets at the leading order accuracy. The obtained leading order cross section at the parton level for $t \bar{t} l^{+} l^{-}+\leq$ 2 jets is $96 \mathrm{fb}$ for $13 \mathrm{TeV}$ LHC and to accommodate the NLO effects we multiplied the cross section with a factor of 1.4 which is the NLO K-factor for $t \bar{t} Z$. Similarly for $t \bar{t} l \nu_{l}+\leq 2$ jets we multiply the leading order parton level cross section $166 \mathrm{fb}$ for $13 \mathrm{TeV}$ LHC with 1.4 which is the $\mathrm{K}$-factor for $t \bar{t} W[61]$.

After the event selection we compare the phase space behavior of the signal events with the background and plot the normalized distributions of the transverse momentum $\left(p_{T}\right)$ of the leading non $\tau$-tagged jet and leading $\tau$-tagged jet along with $\left(E_{T}\right)$ in Fig. 4. Due to the large mass separation between the VLQ and the scalars, the leading jet is expected to be quite hard as the figure shows. In addition, the $\tau$ which comes from the decay of the scalar which is around $300 \mathrm{GeV}$ in mass is also quite hard in $p_{T}$. Thus one can make a quite easy separation of the signal and background events using the distributions shown in Fig. 4. Based on the distributions and to further optimize the signal-to-background ratio, we apply the following kinematic cuts on the final state objects:

$p_{T}\left(j_{1}\right) \geq 200 \mathrm{GeV}, \quad p_{T}\left(\tau_{1}\right) \geq 150 \mathrm{GeV}, \quad E_{T}^{\prime} \geq 150 \mathrm{GeV}$.

With an integrated luminosity of $100 \mathrm{fb}^{-1}$ the cut flow for the signal and background events is shown in Table IV(a). With the above cuts and with $100 \mathrm{fb}^{-1}$ integrated luminosity, the statistical significance for BP1 is quite large $(\sim 12 \sigma)$. We have used $\sqrt{2\left((s+b) \ln \left(1+\frac{s}{b}\right)-s\right)}$ to calculate the significance. Thus BP1 seems to be have VLQs in a mass range which would be very close to the current sensitivity of the LHC run if such a final state is analyzed for VLQs decaying in nonstandard channels as in our model.

\section{B. BP2: $m_{x u_{3}}=1.5 \mathrm{TeV}$ with $3000 \mathrm{fb}^{-1}$}

For BP2 the branching ratios of $x u_{3}$ for the decay modes $h_{2} t, A_{2} t$ and $h_{2}^{+} t$ are around $25.5 \%, 25.5 \%$ and $49 \%$ respectively. The background events and their corresponding cross sections are same as in case of BP1 and we have
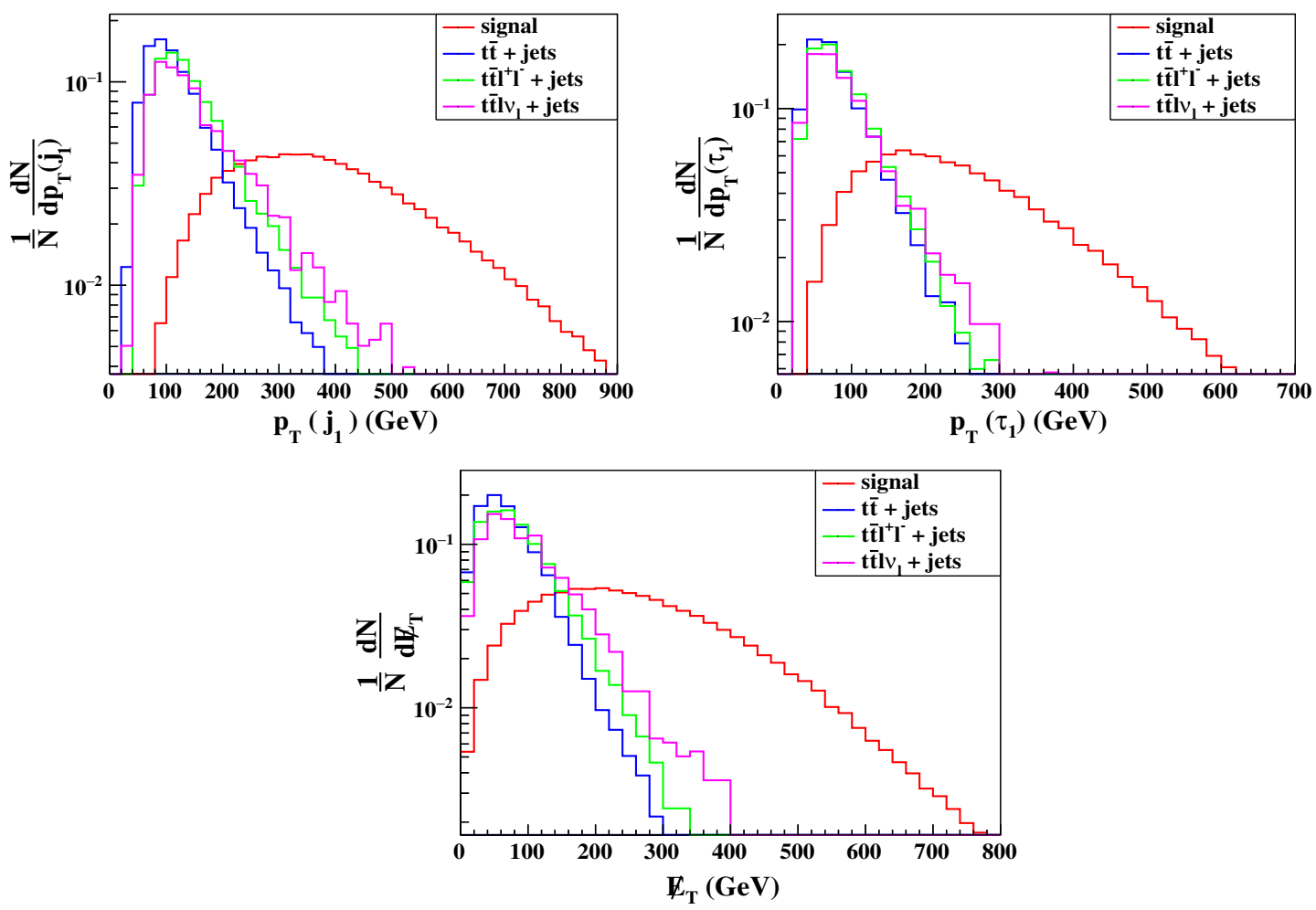

FIG. 4. Normalized distributions for the transverse momentum for the leading non $\tau$-tagged jet $\left(P_{T}\left(j_{1}\right)\right)$, the transverse momentum for the leading $\tau$-tagged jet $\left[P_{T}\left(\tau_{1}\right)\right]$ and the total missing transverse energy $E_{T}$ for BP1 with $m_{x u_{3}}=1 \mathrm{TeV}$. 
TABLE IV. Cut flow table for BP1 with $100 \mathrm{fb}^{-1}, \mathrm{BP} 2$ with $3000 \mathrm{fb}^{-1}$ and BP3 with $3000 \mathrm{fb}^{-1}$ luminosity.

(a)

\begin{tabular}{lcr}
\hline \multicolumn{3}{c}{ BP1: $m_{x u_{3}}=1 \mathrm{TeV}, \mathcal{L}=100 \mathrm{fb}^{-1}$} \\
\hline & \multicolumn{2}{c}{ No. of Events } \\
\cline { 2 - 3 } Cuts & Signal & Background \\
\hline Preselection & 365 & 19677 \\
$p_{T}\left(j_{1}\right) \geq 200 \mathrm{GeV}$ & 320 & 2959 \\
$p_{T}\left(\tau_{1}\right) \geq 150 \mathrm{GeV}$ & 245 & 839 \\
$E_{T} \geq 150 \mathrm{GeV}$ & 188 & 191 \\
\hline \hline
\end{tabular}

(b)

\begin{tabular}{lcc}
\hline \multicolumn{3}{c}{ BP2: $m_{x u_{3}}=1.5 \mathrm{TeV}, \mathcal{L}=3000 \mathrm{fb}^{-1}$} \\
\hline & \multicolumn{2}{c}{ No. of Events } \\
\cline { 2 - 3 } Cuts & Signal & Background \\
\hline Preselection & 455 & 590310 \\
$p_{T}\left(j_{1}\right) \geq 300 \mathrm{GeV}$ & 401 & 28547 \\
$p_{T}\left(\tau_{1}\right) \geq 200 \mathrm{GeV}$ & 307 & 6050 \\
$E_{T} \geq 200 \mathrm{GeV}$ & 245 & 1455 \\
\hline \hline
\end{tabular}

(c)

\begin{tabular}{lcr}
\hline \multicolumn{3}{c}{ BP3: $m_{x u_{3}}=2 \mathrm{TeV}, \mathcal{L}=3000 \mathrm{fb}^{-1}$} \\
\cline { 2 - 3 } & \multicolumn{2}{c}{ No. of Events } \\
\hline Cuts & 86 & Background \\
\hline Preselection & 81 & 856721 \\
$p_{T}\left(j_{1}\right) \geq 350 \mathrm{GeV}$ & 78 & 269121 \\
$p_{T}\left(j_{2}\right) \geq 100 \mathrm{GeV}$ & 58 & 252922 \\
$p_{T}\left(\tau_{1}\right) \geq 150 \mathrm{GeV}$ & 52 & 57285 \\
$E_{T} \geq 200 \mathrm{GeV}$ & 40 & 11571 \\
$M_{\text {eff }} \geq 2.6 \mathrm{TeV}$ & \multicolumn{2}{c}{} \\
\hline \hline
\end{tabular}

used the same preselection criteria on the events (i.e., $\geq 3 j(1 b)+\geq 2 \tau+\geq 1 l$ ) for BP2. The differences between the mass of the VLQ $x u_{3}$ and the masses of scalars $\left(h_{2}, A_{1}\right.$, $h_{1}^{+}$) increase as we go higher in values of the mass of $x u_{3}$ while keeping the mass of the scalars fixed as before. It is worth pointing out here that even if the mass of the scalars are made larger, the decay probabilities of the VLQ do not change much. Therefore the event rates would remain the same, albeit the cut efficiencies would change due to new thresholds for the leading jet and tagged $\tau$. Note that the $b$ quark that will originate from the decay $x u_{3} \rightarrow h_{1}^{+} b$ for a $1.5 \mathrm{TeV} x u_{3}$ will have a large $p_{T}$ compared to the $b$ quark that originates from the decay of a $1 \mathrm{TeV} x u_{3}$. Since the leading non $\tau$-tagged jet is most likely the $b$ jet coming from $h_{1}^{+} b$ mode it will be in general harder in BP2 compared to BP1. Accordingly for $\mathrm{BP} 2$, we have applied the following selection criteria on the final state objects from the reconstructed events to optimize the significance:
$p_{T}\left(j_{1}\right) \geq 300 \mathrm{GeV}, \quad p_{T}\left(\tau_{1}\right) \geq 200 \mathrm{GeV}, \quad E_{T} \geq 200 \mathrm{GeV}$.

Notice after the cut on $p_{T}\left(j_{1}\right)$ to further improve the significance we have also applied cuts with higher values on $p_{T}\left(\tau_{1}\right)$ and $\mathscr{E}_{T}$ compared to the scenario of BP1. For BP2 with $3000 \mathrm{fb}^{-1}$ luminosity the cut flow can be found in the Table IV(b). Using the survived events after the $E_{T}$ cut we get a significance around $6.2 \sigma$ for BP2 with the highluminosity (HL) option at the LHC. With the possibility that the LHC will operate at $\sqrt{s}=14 \mathrm{TeV}$ for the HL option, we estimate that at $14 \mathrm{TeV}$ LHC assuming the same cut efficiencies in Table IV(b), the significance will be around $8.7 \sigma$ with $3000 \mathrm{fb}^{-1}$ of luminosity.

\section{BP3: $m_{x u_{3}}=2 \mathrm{TeV}$ with $3000 \mathrm{fb}^{-1}$}

Finally for the last benchmark, we choose a very heavy mass of $2 \mathrm{TeV}$ for the VLQ. Quite clearly the event rates would suffer from the very small production cross section and if we require two isolated $\tau$-jets then the final events yield becomes extremely low even with an integrated luminosity of $3000 \mathrm{fb}^{-1}$. To counter the suppression due to small production cross section we modify our signal choice to a more inclusive channel given by: $\geq 3$ non $\tau$ tagged jets out of which one is $b$-tagged, at least one $\tau$ tagged jet and at least one lepton in the final state.

As we go higher in the mass of $x u_{3}$ the probability for the jets and the tau leptons for the signal to have higher $p_{T}$ values is more compared to the backgrounds. Hence for BP3 with $m_{x u_{3}}=2 \mathrm{TeV}$ to get a large statistics for the $t \bar{t}+$ jets background, we have generated $p p \rightarrow t \bar{t}+2 j$ events exclusively at the parton level for $13 \mathrm{TeV}$ LHC with the following criteria:

(i) For each event at least one top quark decays leptonically, because at the analysis level we have considered events with at least one lepton in the final state.

(ii) All the jets and leptons satisfy $|\eta|<3.0$ and the angular separation $(\Delta R)$ between all pairs of final state particles are greater than 0.4 (except for leptons where they are separated from each other with minimum angular separation 0.2).

(iii) All the final state objects satisfy $p_{T}>10 \mathrm{GeV}$.

(iv) The two leading jets in $p_{T}$ satisfy $p_{T}\left(j_{1}\right)>$ $250 \mathrm{GeV}$ and $p_{T}\left(j_{2}\right)>100 \mathrm{GeV}$.

With the above cuts the parton level cross section at the leading order accuracy for $p p \rightarrow t \bar{t}+2 j$ with $13 \mathrm{TeV}$ LHC is around $6.18 \mathrm{pb}$. The same events and cross sections as in the case of BP1 has been used for the other two backgrounds $t \bar{t} l^{+} l^{-}+$jets and $t \bar{t} l \nu_{l}+$ jets. Note that with much stronger threshold requirements for the final state jets, we expect that the lesser-order processes involving $t \bar{t}$ and $t \bar{t}+$ $1 j$ would not contribute much, where the extra jet comes from the showering. We then follow the usual procedure of 
using the PythIA showering and DelPHES 3 simulation to generate the final objects from the $p p \rightarrow t \bar{t}+2 j$ process.

For our analysis, we further demand the following set of cuts on our final state events to improve the significance:

$$
\begin{aligned}
& p_{T}\left(j_{1}\right)>350 \mathrm{GeV}, \quad p_{T}\left(j_{2}\right)>100 \mathrm{GeV}, \\
& p_{T}\left(\tau_{1}\right)>150 \mathrm{GeV}, \\
& \mathscr{E}_{T}>200 \mathrm{GeV}, \quad M_{\text {eff }}>2.6 \mathrm{TeV} .
\end{aligned}
$$

Here the effective mass variable $\left(M_{\text {eff }}\right)$ is defined as the scalar sum of all the transverse momenta in an event and is given by

$$
M_{\text {eff }}=\sum_{j \in \text { jets }} p_{T}(j)+\sum_{l \in \text { leptons }} p_{T}(l)+\mathscr{E}_{T} .
$$

The corresponding cut flow can be seen from the Table IV(c) where we have achieved a signal significance of $2 \sigma$ for BP3. As before we again estimate the event rates for the high energy run and find that the expected significance for $14 \mathrm{TeV}$ LHC with $3000 \mathrm{fb}^{-1}$ is around $3.1 \sigma$, if we assume similar cut efficiencies as in Table IV(c).

We plot the significance as a function of luminosity in Fig. 5 for all the benchmark points. It is evident that for BP1 significance of $5 \sigma$ can be achieved with $17.3 \mathrm{fb}^{-1}$ of data, hence with the already existing datasets of $36.1 \mathrm{fb}^{-1}$ of data would be sensitive to this mass range. With high luminosity data the BP2 can be discovered while it is only possible to exclude a $2 \mathrm{TeV}$ VLQ at $2 \sigma$. In fact, an upgrade in LHC energies and higher luminosities would be required to access VLQ signals in such models beyond VLQ mass of $\sim 1.8 \mathrm{TeV}$. This is highlighted in the improvements in significance when $\sqrt{s}=14 \mathrm{TeV}$ is considered for BP-2 and BP-3. Definitive improvements in the sensitivity is also

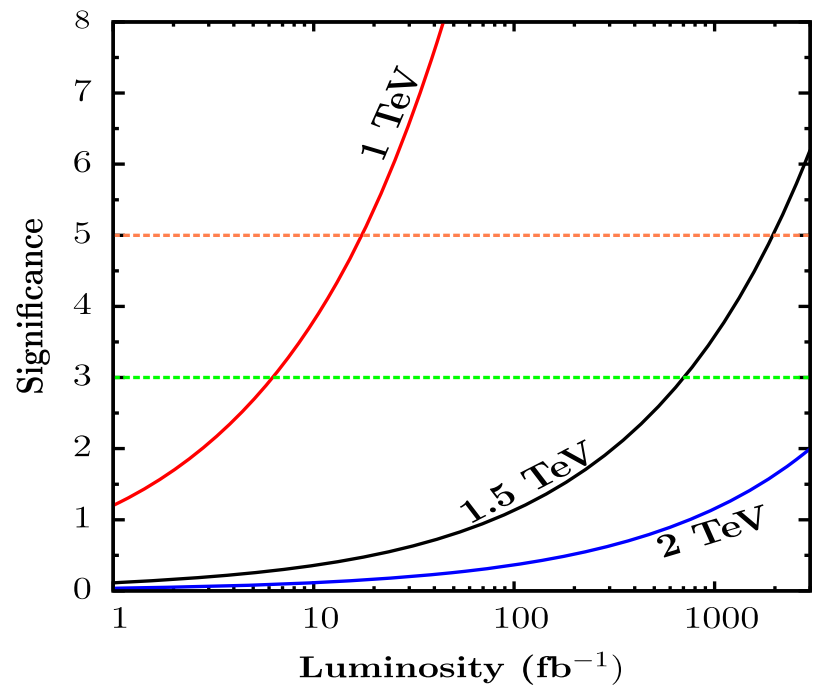

FIG. 5. Significance as a function of luminosity for BP1 (1 TeV), BP2(1.5 TeV) and BP3(2 TeV) for $13 \mathrm{TeV}$ LHC. expected with more sophisticated analysis using boosted studies for the $\tau$ states coming from the decay of the heavily boosted scalars.

\section{CONCLUSION}

In this work we have considered vectorlike quarks in a leptophobic 221 model. Since SM leptons are singlets under the second $S U(2)$, exotic quarks are necessary to cancel triangle anomalies in this model. The exotic quarks become vectorlike after the symmetry breaking of the full symmetry group to the SM gauge group. We discussed a particular mixing pattern between SM quarks and VLQs which avoids tree level FCNC interactions. We also find that the same mixing pattern allows for certain neutral scalars to be flavor-conserving in nature. Two of these neutral scalars and their charged partner are tauphilic in nature. These scalars open up nonstandard decay modes for the VLQs in the model. We studied the collider signatures for pair production of third generation toplike VLQ when it decays to final states with any of the tauphilic scalars and a third generation SM quark. Due to the mass hierarchy in the charged lepton sector, these scalars dominantly decay to tau leptons with more than $99 \%$ probability. We do an analysis for the signal of such VLQs, pair produced at the LHC with $\sqrt{s}=13 \mathrm{TeV}$ through the $\geq 3 j(1 b)+\geq 2 \tau+\geq 1 l$ final state, dictated by the decay properties of the VLQ and the new tauphilic scalars. We use mass threshold driven kinematic selections for the final state objects and show the values of the integrated luminosity required for the discovery of such a toplike VLQ for different benchmark points. We also briefly discuss how the discovery significance improves in case of the $14 \mathrm{TeV}$ HL option of LHC. We find that the amount of data collected till date by the ATLAS and CMS collaborations for $13 \mathrm{TeV}$ LHC is sufficient to confirm or refute the existence of such a scenario for a $1 \mathrm{TeV}$ toplike VLQ. Heavier VLQ masses up to $1.8 \mathrm{TeV}$ would be accessible with the HL option of LHC. This study also highlights an important point of caution for VLQ searches in the standard decay channels carried out at the LHC, that any new physics scenario which may have additional gauge bosons and scalars can alter the VLQ searches in a significant way and therefore alternative channels of search should also be considered, as the VLQ mass limits crucially depend on them [28].

\section{ACKNOWLEDGMENTS}

K. D. would like to thank Tianjun Li for useful discussions, Jyotiranjan Beuria for help regarding SARAH and SPheno, Manuel E. Krauss and Subhadeep Mondal for help regarding SARAH. The work was partially supported by funding available from the Department of Atomic Energy, Government of India, for the Regional Centre for Accelerator-based Particle Physics (RECAPP), HarishChandra Research Institute. The research of K. D. was 
supported in part by the INFOSYS scholarship for senior students at the Harish-Chandra Research Institute. The authors acknowledge the use of the High Performance Scientific Computing facility at RECAPP and HRI.

\section{APPENDIX A: TADPOLE EQUATIONS}

The set of tadpole equations $\left\{\frac{\partial V}{\partial \phi_{1}^{0 r}}=0, \frac{\partial V}{\partial \phi_{2}^{0 r}}=0\right.$, $\left.\frac{\partial V}{\partial \chi^{0 r}}=0, \frac{\partial V}{\partial \chi^{10 r}}=0\right\}$ in terms of $\mu_{1}^{2}, \mu_{2}^{2}, \mu_{3}^{2}, \mu_{4}^{2}$ is given by

$$
\begin{aligned}
\mu_{1}^{2}= & \frac{1}{2\left(v_{1}^{2}-v_{2}^{2}\right)}\left\{\alpha_{1} u^{2} v_{1}^{2}-\alpha_{1} u^{2} v_{2}^{2}-\alpha_{3} u^{2} v_{2}^{2}+2 \lambda_{1} v_{1}^{4}+4 \lambda_{4} v_{2} v_{1}^{3}-4 \lambda_{4} v_{2}^{3} v_{1}-2 \lambda_{1} v_{2}^{4}+\sqrt{2} u v_{3}\left(M_{1} v_{1}-M_{2} v_{2}\right)\right. \\
& \left.+v_{3}^{2}\left(\beta_{1} v_{1}^{2}-\left(\beta_{1}+\beta_{3}\right) v_{2}^{2}\right)\right\}, \\
\mu_{2}^{2}= & \frac{1}{4\left(v_{1}^{2}-v_{2}^{2}\right)}\left\{2 \alpha_{2} u^{2} v_{1}^{2}+\alpha_{3} u^{2} v_{2} v_{1}-2 \alpha_{2} u^{2} v_{2}^{2}+2 \lambda_{4} v_{1}^{4}+8 \lambda_{2} v_{2} v_{1}^{3}+4 \lambda_{3} v_{2} v_{1}^{3}-8 \lambda_{2} v_{2}^{3} v_{1}-4 \lambda_{3} v_{2}^{3} v_{1}-2 \lambda_{4} v_{2}^{4}\right. \\
& \left.+\sqrt{2} u v_{3}\left(M_{2} v_{1}-M_{1} v_{2}\right)+v_{3}^{2}\left(2 \beta_{2}\left(v_{1}^{2}-v_{2}^{2}\right)+\beta_{3} v_{1} v_{2}\right)\right\}, \\
\mu_{3}^{2}= & \frac{1}{2 v_{3}}\left\{\sqrt{2} u\left(M_{1} v_{1}+M_{2} v_{2}\right)+v_{3}\left(\rho_{3} u^{2}+\beta_{1} v_{1}^{2}+4 \beta_{2} v_{1} v_{2}+\left(\beta_{1}+\beta_{3}\right) v_{2}^{2}\right)+2 \rho_{1} v_{3}^{3}\right\}, \\
\mu_{4}^{2}= & \frac{1}{2 u}\left\{v_{1}\left(\sqrt{2} M_{1} v_{3}+4 \alpha_{2} u v_{2}\right)+\sqrt{2} M_{2} v_{2} v_{3}+u\left(2 \rho_{2} u^{2}+\left(\alpha_{1}+\alpha_{3}\right) v_{2}^{2}+\rho_{3} v_{3}^{2}\right)+\alpha_{1} u v_{1}^{2}\right\} .
\end{aligned}
$$

\section{APPENDIX B: MASS MATRICES FOR THE SCALAR SECTOR}

\section{1. $C P$ even scalars}

The components of the $C P$ even scalar sector mass square matrix $\left(M_{S}^{2}\right)$ in the $\left(\phi_{1}^{0 r}, \phi_{2}^{0 r}, \chi^{\prime 0 r}, \chi^{0 r}\right)$ basis is given by

$$
\begin{aligned}
& \left(M_{S}^{2}\right)_{11}=\frac{1}{2\left(v_{1}^{2}-v_{2}^{2}\right)}\left\{v_{2}\left(\sqrt{2} M_{2} u v_{3}+\alpha_{3} u^{2} v_{2}-4\left(2 \lambda_{2}+\lambda_{3}\right) v_{2}^{3}\right)-v_{1}\left(\sqrt{2} M_{1} u v_{3}+8 \lambda_{4} v_{2}^{3}\right)\right. \\
& \left.+\beta_{3} v_{2}^{2} v_{3}^{2}+4 \lambda_{1} v_{1}^{4}+8 \lambda_{4} v_{2} v_{1}^{3}+4\left(-\lambda_{1}+2 \lambda_{2}+\lambda_{3}\right) v_{2}^{2} v_{1}^{2}\right\} \\
& \left(M_{S}^{2}\right)_{12}=\frac{1}{2\left(v_{1}^{2}-v_{2}^{2}\right)}\left\{-v_{1}\left(\sqrt{2} M_{2} u v_{3}+\alpha_{3} u^{2} v_{2}+4\left(\lambda_{1}+2 \lambda_{2}+\lambda_{3}\right) v_{2}^{3}\right)+\sqrt{2} M_{1} u v_{2} v_{3}\right. \\
& \left.-\beta_{3} v_{2} v_{3}^{2} v_{1}+4 \lambda_{4} v_{1}^{4}+4\left(\lambda_{1}+2 \lambda_{2}+\lambda_{3}\right) v_{2} v_{1}^{3}-4 \lambda_{4} v_{2}^{4}\right\} \\
& \left(M_{S}^{2}\right)_{13}=\frac{M_{1} v_{3}}{\sqrt{2}}+u\left(\alpha_{1} v_{1}+2 \alpha_{2} v_{2}\right) \\
& \left(M_{S}^{2}\right)_{14}=\frac{M_{1} u}{\sqrt{2}}+v_{3}\left(\beta_{1} v_{1}+2 \beta_{2} v_{2}\right) \\
& \left(M_{S}^{2}\right)_{22}=\frac{1}{2\left(v_{1}^{2}-v_{2}^{2}\right)}\left\{-v_{1}\left(\sqrt{2} M_{1} u v_{3}+8 \lambda_{4} v_{2}^{3}\right)+\sqrt{2} M_{2} u v_{2} v_{3}+\beta_{3} v_{3}^{2} v_{1}^{2}+4\left(2 \lambda_{2}+\lambda_{3}\right) v_{1}^{4}\right. \\
& \left.+8 \lambda_{4} v_{2} v_{1}^{3}-4 \lambda_{1} v_{2}^{4}+v_{1}^{2}\left(\alpha_{3} u^{2}+4\left(\lambda_{1}-2 \lambda_{2}-\lambda_{3}\right) v_{2}^{2}\right)\right\} \\
& \left(M_{S}^{2}\right)_{23}=\frac{M_{2} v_{3}}{\sqrt{2}}+u\left(2 \alpha_{2} v_{1}+\left(\alpha_{1}+\alpha_{3}\right) v_{2}\right) \\
& \left(M_{S}^{2}\right)_{24}=\frac{M_{2} u}{\sqrt{2}}+v_{3}\left(2 \beta_{2} v_{1}+\left(\beta_{1}+\beta_{3}\right) v_{2}\right) \\
& \left(M_{S}^{2}\right)_{33}=\frac{1}{2 u}\left\{4 \rho_{2} u^{3}-\sqrt{2} v_{3}\left(M_{1} v_{1}+M_{2} v_{2}\right)\right\} \\
& \left(M_{S}^{2}\right)_{34}=\frac{M_{1} v_{1}}{\sqrt{2}}+\frac{M_{2} v_{2}}{\sqrt{2}}+\rho_{3} u v_{3} \\
& \left(M_{S}^{2}\right)_{44}=\frac{1}{2 v_{3}}\left\{4 \rho_{1} v_{3}^{3}-\sqrt{2} u\left(M_{1} v_{1}+M_{2} v_{2}\right)\right\}
\end{aligned}
$$




\section{2. $\boldsymbol{C P}$ odd scalars}

The components of the $C P$ odd scalar sector mass square matrix $\left(M_{P}^{2}\right)$ in the $\left(\phi_{1}^{0 i}, \phi_{2}^{0 i}, \chi^{10 i}, \chi^{0 i}\right)$ basis is given by

$$
\begin{aligned}
\left(M_{P}^{2}\right)_{11}= & \frac{1}{2\left(v_{1}^{2}-v_{2}^{2}\right)}\left\{\sqrt{2} u v_{3}\left(M_{2} v_{2}-M_{1} v_{1}\right)\right. \\
& \left.+v_{2}^{2}\left(\alpha_{3} u^{2}-4\left(2 \lambda_{2}-\lambda_{3}\right)\left(v_{1}^{2}-v_{2}^{2}\right)\right)+\beta_{3} v_{3}^{2} v_{2}^{2}\right\} \\
\left(M_{P}^{2}\right)_{12}= & \frac{1}{2\left(v_{1}^{2}-v_{2}^{2}\right)}\left\{\sqrt{2} u v_{3}\left(M_{2} v_{1}-M_{1} v_{2}\right)\right. \\
& \left.+v_{1} v_{2}\left(\alpha_{3} u^{2}-4\left(2 \lambda_{2}-\lambda_{3}\right)\left(v_{1}^{2}-v_{2}^{2}\right)\right)+\beta_{3} v_{1} v_{2} v_{3}^{2}\right\} \\
\left(M_{P}^{2}\right)_{13}= & -\frac{M_{1} v_{3}}{\sqrt{2}} \\
\left(M_{P}^{2}\right)_{14}= & \frac{M_{1} u}{\sqrt{2}} \\
\left(M_{P}^{2}\right)_{22}= & \frac{1}{2\left(v_{1}^{2}-v_{2}^{2}\right)}\left\{\sqrt{2} u v_{3}\left(M_{2} v_{2}-M_{1} v_{1}\right)\right. \\
& \left.+v_{1}^{2}\left(\alpha_{3} u^{2}-4\left(2 \lambda_{2}-\lambda_{3}\right)\left(v_{1}^{2}-v_{2}^{2}\right)\right)+\beta_{3} v_{3}^{2} v_{1}^{2}\right\} \\
\left(M_{P}^{2}\right)_{23}= & \frac{M_{2} v_{3}}{\sqrt{2}} \\
\left(M_{P}^{2}\right)_{24}= & -\frac{M_{2} u}{\sqrt{2}} \\
\left(M_{P}^{2}\right)_{33}= & -\frac{v_{3}\left(M_{1} v_{1}+M_{2} v_{2}\right)}{\sqrt{2} u} \\
\left(M_{P}^{2}\right)_{34}= & \frac{M_{1} v_{1}+M_{2} v_{2}}{\sqrt{2}} \\
\left(M_{P}^{2}\right)_{44}= & -\frac{u\left(M_{1} v_{1}+M_{2} v_{2}\right)}{\sqrt{2} v_{3}}
\end{aligned}
$$

\section{Charged scalars}

The mass square matrix for the charged scalars $\left(M_{C}^{2}\right)$ in the $\left(\phi_{1}^{+}, \phi_{2}^{+}, \chi^{\prime+}, \chi^{+}\right)$basis is given by

$$
\begin{aligned}
\left(M_{C}^{2}\right)_{11}= & \frac{1}{2\left(v_{1}^{2}-v_{2}^{2}\right)}\left\{u\left(\sqrt{2} v_{3}\left(M_{2} v_{2}-M_{1} v_{1}\right)+\alpha_{3} u v_{2}^{2}\right)\right. \\
& \left.+\beta_{3} v_{1}^{2} v_{3}^{2}\right\} \\
\left(M_{C}^{2}\right)_{12}= & \frac{1}{2\left(v_{1}^{2}-v_{2}^{2}\right)}\left\{u\left(\sqrt{2} v_{3}\left(M_{2} v_{1}-M_{1} v_{2}\right)+\alpha_{3} u v_{1} v_{2}\right)\right. \\
& \left.+\beta_{3} v_{1} v_{2} v_{3}^{2}\right\} \\
\left(M_{C}^{2}\right)_{13}= & -\frac{M_{2} v_{3}}{\sqrt{2}}-\frac{1}{2} \alpha_{3} u v_{2} \\
\left(M_{C}^{2}\right)_{14}= & \frac{M_{1} u}{\sqrt{2}}-\frac{1}{2} \beta_{3} v_{1} v_{3} \\
\left(M_{C}^{2}\right)_{22}= & \frac{1}{2\left(v_{1}^{2}-v_{2}^{2}\right)}\left\{u\left(\sqrt{2} v_{3}\left(M_{2} v_{2}-M_{1} v_{1}\right)+\alpha_{3} u v_{1}^{2}\right)\right. \\
& \left.+\beta_{3} v_{2}^{2} v_{3}^{2}\right\} \\
\left(M_{C}^{2}\right)_{23}= & \frac{M_{1} v_{3}}{\sqrt{2}-\frac{1}{2} \alpha_{3} u v_{1}} \\
\left(M_{C}^{2}\right)_{24}= & -\frac{M_{2} u}{\sqrt{2}}-\frac{1}{2} \beta_{3} v_{2} v_{3} \\
\left(M_{C}^{2}\right)_{33}= & \frac{1}{2}\left(\alpha_{3}\left(v_{1}^{2}-v_{2}^{2}\right)-\frac{\sqrt{2} v_{3}\left(M_{1} v_{1}+M_{2} v_{2}\right)}{u}\right) \\
\left(M_{C}^{2}\right)_{34}= & \frac{M_{2} v_{1}+M_{1} v_{2}}{\sqrt{2}} \\
\left(M_{C}^{2}\right)_{44}= & \frac{1}{2 v_{3}}\left\{\beta_{3}\left(v_{1}^{2}-v_{2}^{2}\right) v_{3}-\sqrt{2} u\left(M_{1} v_{1}+M_{2} v_{2}\right)\right\} \\
&
\end{aligned}
$$

[1] O. Eberhardt, G. Herbert, H. Lacker, A. Lenz, A. Menzel, U. Nierste, and $\mathrm{M}$. Wiebusch, Impact of a Higgs Boson at a Mass of $126 \mathrm{GeV}$ on the Standard Model with Three and Four Fermion Generations, Phys. Rev. Lett. 109, 241802 (2012).

[2] A. M. Sirunyan et al. (CMS Collaboration), Search for vector-like T and B quark pairs in final states with leptons at $\sqrt{s}=13$ TeV, J. High Energy Phys. 08 (2018) 177.

[3] A. M. Sirunyan et al. (CMS Collaboration), Search for pair production of vector-like quarks in the $\mathrm{bW} \overline{\mathrm{b}} \mathrm{W}$ channel from proton-proton collisions at $\sqrt{s}=13 \mathrm{TeV}$, Phys. Lett. B 779, 82 (2018).

[4] M. Aaboud et al. (ATLAS Collaboration), Search for pair- and single-production of vector-like quarks in final states with at least one $Z$ boson decaying into a pair of electrons or muons in $p p$ collision data collected with the
ATLAS detector at $\sqrt{s}=13 \mathrm{TeV}$, Phys. Rev. D 98, 112010 (2018).

[5] M. Aaboud et al. (ATLAS Collaboration), Search for pair production of heavy vector-like quarks decaying into high$p_{T} W$ bosons and top quarks in the lepton-plus-jets final state in $p p$ collisions at $\sqrt{s}=13 \mathrm{TeV}$ with the ATLAS detector, J. High Energy Phys. 08 (2018) 048.

[6] M. Aaboud et al. (ATLAS Collaboration), Search for pair production of up-type vector-like quarks and for four-topquark events in final states with multiple $b$-jets with the ATLAS detector, J. High Energy Phys. 07 (2018) 089.

[7] M. Aaboud et al. (ATLAS Collaboration), Search for pair production of heavy vector-like quarks decaying to high- $\mathrm{p}_{T}$ $\mathrm{W}$ bosons and $\mathrm{b}$ quarks in the lepton-plus-jets final state in pp collisions at $\sqrt{s}=13 \mathrm{TeV}$ with the ATLAS detector, J. High Energy Phys. 10 (2017) 141. 
[8] A. M. Sirunyan et al. (CMS Collaboration), Search for single production of vector-like quarks decaying to $\mathrm{a} b$ quark and a Higgs boson, J. High Energy Phys. 06 (2018) 031.

[9] A. M. Sirunyan et al. (CMS Collaboration), Search for single production of a vector-like $\mathrm{T}$ quark decaying to a $\mathrm{Z}$ boson and a top quark in proton-proton collisions at $\sqrt{s}=13 \mathrm{TeV}$, Phys. Lett. B 781, 574 (2018).

[10] ATLAS Collaboration, Search for single production of vector-like quarks decaying into $W b$ in $p p$ collisions at $\sqrt{s}=13 \mathrm{TeV}$ with the ATLAS detector, Report No. ATLAS-CONF-2016-072, 2016, https://atlas.web.cern.ch/ Atlas/GROUPS/PHYSICS/CONFNOTES/ATLAS-CONF2016-072/.

[11] J. A. Aguilar-Saavedra, Pair production of heavy $Q=2 / 3$ singlets at LHC, Phys. Lett. B 625, 234 (2005).

[12] J. A. Aguilar-Saavedra, Identifying top partners at LHC, J. High Energy Phys. 11 (2009) 030.

[13] G. Cacciapaglia, A. Deandrea, D. Harada, and Y. Okada, Bounds and decays of new heavy vector-like top partners, J. High Energy Phys. 11 (2010) 159.

[14] Y. Okada and L. Panizzi, LHC signatures of vector-like quarks, Adv. High Energy Phys. 2013, 1 (2013).

[15] A. De Simone, O. Matsedonskyi, R. Rattazzi, and A. Wulzer, A first top partner hunter's guide, J. High Energy Phys. 04 (2013) 004.

[16] J. A. Aguilar-Saavedra, R. Benbrik, S. Heinemeyer, and M. Pérez-Victoria, Handbook of vectorlike quarks: Mixing and single production, Phys. Rev. D 88, 094010 (2013).

[17] S. A. R. Ellis, R. M. Godbole, S. Gopalakrishna, and J. D. Wells, Survey of vector-like fermion extensions of the Standard Model and their phenomenological implications, J. High Energy Phys. 09 (2014) 130.

[18] K. Hsieh, K. Schmitz, J.-H. Yu, and C. P. Yuan, Global analysis of general $\mathrm{SU}(2) \times \mathrm{SU}(2) \times \mathrm{U}(1)$ models with precision data, Phys. Rev. D 82, 035011 (2010).

[19] K. Das, T. Li, S. Nandi, and S. K. Rai, Diboson excesses in an anomaly free leptophobic left-right model, Phys. Rev. D 93, 016006 (2016).

[20] P. Langacker, The physics of heavy $Z^{\prime}$ gauge bosons, Rev. Mod. Phys. 81, 1199 (2009).

[21] J. Kearney, A. Pierce, and J. Thaler, Top partner probes of extended Higgs sectors, J. High Energy Phys. 08 (2013) 130.

[22] J. Kearney, A. Pierce, and J. Thaler, Exotic top partners and little Higgs, J. High Energy Phys. 10 (2013) 230.

[23] D. Karabacak, S. Nandi, and S. K. Rai, New signal for singlet Higgs and vector-like quarks at the LHC, Phys. Lett. B 737, 341 (2014).

[24] J. Serra, Beyond the minimal top partner decay, J. High Energy Phys. 09 (2015) 176.

[25] A. Anandakrishnan, J. H. Collins, M. Farina, E. Kuflik, and M. Perelstein, Odd top partners at the LHC, Phys. Rev. D 93, 075009 (2016).

[26] S. Banerjee, D. Barducci, G. Bélanger, and C. Delaunay, Implications of a high-mass diphoton resonance for heavy quark searches, J. High Energy Phys. 11 (2016) 154.

[27] A. Arhrib, R. Benbrik, S. King, B. Manaut, S. Moretti, and C. Un, Phenomenology of 2HDM with vectorlike quarks, Phys. Rev. D 97, 095015 (2018).
[28] B. A. Dobrescu and F. Yu, Exotic signals of vectorlike quarks, J. Phys. G 45, 08 (2016).

[29] J. A. Aguilar-Saavedra, D. E. López-Fogliani, and C. Muñoz, Novel signatures for vector-like quarks, J. High Energy Phys. 06 (2017) 095.

[30] M. Chala, Direct bounds on heavy toplike quarks with standard and exotic decays, Phys. Rev. D 96, 015028 (2017).

[31] S. Moretti, D. O'Brien, L. Panizzi, and H. Prager, Production of extra quarks decaying to dark matter beyond the narrow width approximation at the LHC, Phys. Rev. D 96, 035033 (2017).

[32] M. Chala, R. Gröber, and M. Spannowsky, Searches for vector-like quarks at future colliders and implications for composite Higgs models with dark matter, J. High Energy Phys. 03 (2018) 040.

[33] N. Bizot, G. Cacciapaglia, and T. Flacke, Common exotic decays of top partners, J. High Energy Phys. 06 (2018) 065.

[34] J. H. Kim and I. M. Lewis, Loop induced single top partner production and decay at the LHC, J. High Energy Phys. 05 (2018) 095.

[35] B. N. Grossmann, B. McElrath, S. Nandi, and S. K. Rai, Hidden extra U(1) at the electroweak/TeV scale, Phys. Rev. D 82, 055021 (2010).

[36] A. Joglekar and J. L. Rosner, Searching for signatures of $E_{6}$, Phys. Rev. D 96, 015026 (2017).

[37] K. Das, T. Li, S. Nandi, and S. K. Rai, New signals for vector-like down-type quark in $U(1)$ of $E_{6}$, Eur. Phys. J. C 78, 35 (2018).

[38] J. A. Aguilar-Saavedra, Effects of mixing with quark singlets, Phys. Rev. D 67, 035003 (2003).

[39] G. Cacciapaglia, A. Deandrea, L. Panizzi, N. Gaur, D. Harada, and Y. Okada, Heavy vector-like top partners at the LHC and flavour constraints, J. High Energy Phys. 03 (2012) 070.

[40] J. A. Aguilar-Saavedra, Mixing with vector-like quarks: Constraints and expectations, EPJ Web Conf. 60, 16012 (2013).

[41] A. K. Alok, S. Banerjee, D. Kumar, and S. Uma Sankar, Flavor signatures of isosinglet vector-like down quark model, Nucl. Phys. B906, 321 (2016).

[42] A. K. Alok, S. Banerjee, D. Kumar, S. U. Sankar, and D. London, New-physics signals of a model with a vectorsinglet up-type quark, Phys. Rev. D 92, 013002 (2015).

[43] C.-Y. Chen, S. Dawson, and E. Furlan, Vectorlike fermions and Higgs effective field theory revisited, Phys. Rev. D 96, 015006 (2017).

[44] P. Langacker and D. London, Mixing between ordinary and exotic fermions, Phys. Rev. D 38, 886 (1988).

[45] R. N. Mohapatra and J. C. Pati, A natural left-right symmetry, Phys. Rev. D 11, 2558 (1975).

[46] R. N. Mohapatra and J. C. Pati, Left-right gauge symmetry and an isoconjugate model of $C P$ violation, Phys. Rev. D 11, 566 (1975).

[47] G. Senjanovic and R. N. Mohapatra, Exact left-right symmetry and spontaneous violation of parity, Phys. Rev. D 12, 1502 (1975).

[48] G. Senjanovic, Spontaneous breakdown of parity in a class of gauge theories, Nucl. Phys. B153, 334 (1979). 
[49] R. N. Mohapatra and G. Senjanovic, Neutrino Mass and Spontaneous Parity Violation, Phys. Rev. Lett. 44, 912 (1980).

[50] R. N. Mohapatra and G. Senjanovic, Neutrino masses and mixings in gauge models with spontaneous parity violation, Phys. Rev. D 23, 165 (1981).

[51] G. Aad et al. (ATLAS Collaboration), Search for high-mass diboson resonances with boson-tagged jets in proton-proton collisions at $\sqrt{s}=8 \mathrm{TeV}$ with the ATLAS detector, J. High Energy Phys. 12 (2015) 055.

[52] N. G. Deshpande, J. F. Gunion, B. Kayser, and F. I. Olness, Left-right symmetric electroweak models with triplet Higgs, Phys. Rev. D 44, 837 (1991).

[53] J. F. Gunion, J. Grifols, A. Mendez, B. Kayser, and F. I. Olness, Higgs bosons in left-right symmetric models, Phys. Rev. D 40, 1546 (1989).

[54] M. Aaboud et al. (ATLAS Collaboration), Search for new phenomena in dijet events using $37 \mathrm{fb}^{-1}$ of $p p$ collision data collected at $\sqrt{s}=13 \mathrm{TeV}$ with the ATLAS detector, Phys. Rev. D 96, 052004 (2017).

[55] M. Aaboud et al. (ATLAS Collaboration), Search for new high-mass phenomena in the dilepton final state using $36 \mathrm{fb}^{-1}$ of proton-proton collision data at $\sqrt{s}=13 \mathrm{TeV}$ with the ATLAS detector, J. High Energy Phys. 10 (2017) 182.

[56] J. Erler, P. Langacker, S. Munir, and E. Rojas, Improved constraints on Z-prime bosons from electroweak precision data, J. High Energy Phys. 08 (2009) 017.

[57] R. D. Ball et al. (NNPDF Collaboration), Parton distributions for the LHC Run II, J. High Energy Phys. 04 (2015) 040 .

[58] J. Abdallah et al. (DELPHI Collaboration), Searches for neutral higgs bosons in extended models, Eur. Phys. J. C 38, 1 (2004).
[59] F. Staub, SARAH 4: A tool for (not only SUSY) model builders, Comput. Phys. Commun. 185, 1773 (2014).

[60] W. Porod and F. Staub, SPheno 3.1: Extensions including flavour, $C P$-phases and models beyond the MSSM, Comput. Phys. Commun. 183, 2458 (2012).

[61] J. Alwall, R. Frederix, S. Frixione, V. Hirschi, F. Maltoni, O. Mattelaer, H.-S. Shao, T. Stelzer, P. Torrielli, and M. Zaro, The automated computation of tree-level and next-toleading order differential cross sections, and their matching to parton shower simulations, J. High Energy Phys. 07 (2014) 079.

[62] T. Sjostrand, S. Mrenna, and P.Z. Skands, PYTHIA 6.4 physics and manual, J. High Energy Phys. 05 (2006) 026.

[63] J. de Favereau, C. Delaere, P. Demin, A. Giammanco, V. Lemaître, A. Mertens, and M. Selvaggi (DELPHES 3 Collaboration), DELPHES 3, A modular framework for fast simulation of a generic collider experiment, J. High Energy Phys. 02 (2014) 057.

[64] M. Cacciari, G. P. Salam, and G. Soyez, FastJet user manual, Eur. Phys. J. C 72, 1896 (2012).

[65] E. Conte, B. Fuks, and G. Serret, MadAnalysis 5, a userfriendly framework for collider phenomenology, Comput. Phys. Commun. 184, 222 (2013).

[66] ATLAS Collaboration, Reconstruction, energy calibration, and identification of hadronically decaying tau leptons in the ATLAS experiment for run-2 of the LHC, Report No. ATL-PHYS-PUB-2015-045, 2015, https://cds.cern.ch/ record/2064383.

[67] M. Czakon and A. Mitov, Top++: A program for the calculation of the top-pair cross-section at hadron colliders, Comput. Phys. Commun. 185, 2930 (2014). 